\title{
ON EXISTENCE OF WEAK SOLUTION TO A MODEL DESCRIBING INCOMPRESSIBLE MIXTURES WITH THERMAL DIFFUSION CROSS EFFECTS
}

\author{
MIROSLAV BULÍČEK AND JAROSLAV HAVRDA
}

\begin{abstract}
We present a model describing unsteady flows of a heat conducting mixture composed from $L$ constituents in two and three dimensional bounded domain. We assume that the flow of the mixture is described only by the barycentric velocity, and that the fluid is nonNewtonian. In addition, we assume that the diffusion flux depends also on the temperature gradient, describing the Soret effect, and that the heat flux depends also on the chemical potentials gradient, describing the Dufour effect. We briefly show under which assumptions on the constitutive equations the model obeys the first and the second laws of thermodynamics and for a large class of physically well-motivated constitutive relations we establish the existence of a weak solution. For simplicity we restrict ourselves only onto the linear models, i.e., the diffusion and the heat flux depend linearly on the temperature and chemical potentials gradients.
\end{abstract}

\section{INTRODUCTION}

We deal with a class of models for incompressible fluid mixture, where the diffusion of each constituent and also the heat flux are driven by the chemical potentials gradient and by the temperature gradient as well. Our main goals are to identify the possible structure of the fluxes that is in agreement with the laws of thermodynamics and to start the mathematical exploitation of such models, which means in our case to establish the existence of a weak solution. We want to extend the existing mathematical theory developed in $[6,25,26,27]$ (for the analysis of compressible models, we refer the interested reader to $[1,11]$ ) in many directions. First, in $[6]$ the authors considered the simplified model of a mixture with only two constituents neglecting all thermal effects. Second, the series of papers [25, 26, 27] deals even with the ionized mixtures with thermal effect but is not capable to cover the Soret and the Dufour effects. In addition in this series the models does not fulfill the second law of thermodynamics. All these drawbacks from papers mentioned above are not presented in the model studied here and as the main improvement in comparison to previous works can be understood the use of the chemical potentials directly in the model and a priori estimates in view of the thermodynamics of the model. Primary, we do not want to derive a new classes of models for fluid mixtures but to present a new view of rather standard models ${ }^{1}$. It is also worth of noticing that the model presented here is compatible with the so-called GENERIC framework and in fact it extends the models obtained in [30] and give the answer on the question about the existence of the weak solution. For other relevant works inspired by GENERIC, we refer to [15] or [29].

At the very beginning, we would like to emphasize that the basis of the paper is the use of the barycentric velocity as the only macroscopic velocity, see e.g. [9, 23] for more complete description of such an assumption. We use the linear treatment of the chemical reactions, as used e.g. in [16] which can be also understood as a good summary of the theory of linear irreversible thermodynamics and we also refer to [13] for particular examples of diffusion laws for the chemical

2010 Mathematics Subject Classification. 76D03,35Q79,35Q35,80A32,80A17,76V05.

Key words and phrases. mixture, incompressible fluid, non-Newtonian fluids, heat conducting fluid, Soret effect, Dufour effect, cross-effects, existence of a weak solution.

The authors thank the project 201/09/0917 financed by Czech Science Foundation (GAČR) and the project 309811/2011 financed by the Grant Agency of Charles University. The support of the Karel Janeček Foundation is also acknowledged. M. Bulíček is a researcher in the University Centre for Mathematical Modelling, Applied Analysis and Computational Mathematics (Math MAC).

${ }^{1}$ For alternative models see $[8,17]$. 
concentrations. A similar treatment of chemical reactions appears already in [22] and the gradient structure of chemical reactions is emphasized in [20], where in contrast to this paper the free energy approach is used.

The structure of the paper is following. In Section 2 we formulate the model we are interested in and we also specify all necessary conditions that follow from the laws of thermodynamics. Further, we also specify precisely the assumptions on the constitutive equations and give several physically well-motivated examples fulfilling such assumptions. In Section 3, we introduce the function spaces and auxiliary results, formulate the definition of a weak solution and finally state main theorems of the paper. The last two sections are devoted to the proofs of the theorems.

\section{Formulation OF THE MODEL}

This section is devoted to the description of a general physical background of the model and we also discuss here what are the necessary assumptions on the data and on the model to have its physical consistency. For much more sophisticated discussion, we refer here to [21, 24, 28], where the rational thermodynamics for mixtures is explained in more details, see also $[12,14,18]$.

Hence for a given time interval $I:=(0, T)$ and given Lipschitz bounded domain $\Omega \subset \mathbb{R}^{d}$ with dimension $d=2,3$, we denote $Q:=I \times \Omega$ and consider that the flow takes place in $Q$. First, we assume that a mixture consists from $L$ constituents, where $L \in \mathbb{N}$ is given. Each constituent is described by its own density $\rho_{i}: Q \rightarrow \mathbb{R}_{+}$and the velocity $\boldsymbol{v}_{i}: Q \rightarrow \mathbb{R}^{d}$, where $i=1, \ldots, L$. Consequently the balance of mass for each constituent takes the form

$$
\left(\rho_{i}\right)_{, t}+\operatorname{div}\left(\rho_{i} \boldsymbol{v}_{i}\right)=r_{i} \quad \text { in } Q, \text { for all } i=1, \ldots, L,
$$

where $r_{i}$ denotes the production rate of the $i$-th constituent which can depend on a macroscopic quantities (see below for precise assumption). For simplicity we denote the vector $\mathfrak{r}:=\left(r_{1}, \ldots, r_{L}\right)$. Then we introduce the total density of the fluid $\varrho: Q \rightarrow \mathbb{R}_{+}$and the barycentric velocity $\boldsymbol{v}: Q \rightarrow$ $\mathbb{R}^{d}$ as

$$
\varrho:=\sum_{i=1}^{L} \rho_{i}, \quad \boldsymbol{v}:=\frac{\sum_{i=1}^{L} \rho_{i} \boldsymbol{v}_{i}}{\varrho} .
$$

The key simplification and the heart of the modelling here is that we assume in the paper that the only macroscopic velocity is the barycentric one and we model all balance laws in terms of $\boldsymbol{v}$ instead of taking into account all $\boldsymbol{v}_{i}$ 's. Thus, taking the sum in $(2.1)$ with respect to $i=1, \ldots, L$, we observe the balance of the total mass

$$
\varrho_{, t}+\operatorname{div}(\varrho \boldsymbol{v})=\sum_{i=1}^{L} r_{i} \quad \text { in } Q .
$$

Second, we replace $\boldsymbol{v}_{i}$ in (2.1) by the barycentric velocity $\boldsymbol{v}$ and model the error, i.e., the quantity $\left(\rho_{i} \boldsymbol{v}_{i}-\rho_{i} \boldsymbol{v}\right)$ as a flux (diffusion) $\mathbf{q}_{\mathfrak{c}}:=\left(\mathbf{q}_{\mathfrak{c}}^{1}, \ldots, \mathbf{q}_{\mathfrak{c}}^{L}\right): Q \rightarrow \mathbb{R}^{d \times L}$, which will be specified later. Thus, (2.1) reduces to

$$
\left(\varrho c_{i}\right)_{, t}+\operatorname{div}\left(\varrho c_{i} \boldsymbol{v}+\mathbf{q}_{\mathfrak{c}}^{i}\right)=r_{i} \quad \text { in } Q, \text { for all } i=1, \ldots, L,
$$

where the vector of concentrations $\mathfrak{c}=\left(c_{1}, \ldots, c_{L}\right)$ is defined as

$$
c_{i}:=\frac{\rho_{i}}{\varrho}, \quad i=1, \ldots, L
$$

Next, the balance of linear momentum takes the form (we omit the external body forces for simplicity)

$$
(\varrho \boldsymbol{v})_{, t}+\operatorname{div}(\varrho \boldsymbol{v} \otimes \boldsymbol{v})-\operatorname{div} \mathbf{T}=\mathbf{0} \quad \text { in } Q .
$$

Here $\mathbf{T}: Q \rightarrow \mathbb{R}^{d \times d}$ is the Cauchy stress tensor (that is supposed to be symmetric due to the balance of angular momentum). Next, we introduce the balance of the total energy $E=\frac{\varrho|v|^{2}}{2}+\varrho e$, which is given as the sum of the kinetic energy and the internal energy $e: Q \rightarrow \mathbb{R}_{+}$,

$$
E_{, t}+\operatorname{div}(E \boldsymbol{v})-\operatorname{div}(\mathbf{T} \boldsymbol{v})+\operatorname{div} \mathbf{q}_{e}=0 \quad \text { in } Q
$$


where we denoted $\mathbf{q}_{e}: Q \rightarrow \mathbb{R}^{d}$ the flux of the internal energy $e$ and we assume that there are no volume sources of the global energy. The final simplification we make, is the assumption that the mixture as a whole is homogeneous, i.e., we assume that $\varrho=$ const in $Q$ and for simplicity but without loss of generality we set $\varrho \equiv 1$ in what follows. Consequently, we also require the incompressibility constraint

$$
\operatorname{div} \boldsymbol{v}=0 \quad \text { in } Q,
$$

and we assume that the Cauchy stress $\mathbf{T}$ can be decomposed as

$$
\mathbf{T}:=-p \mathbf{I}+\mathbf{S},
$$

where $p: Q \rightarrow \mathbb{R}$ denotes the mean normal stress (the pressure) and $\mathbf{S}: Q \rightarrow \mathbb{R}^{d \times d}$ is the constitutively determined part of the Cauchy stress.

To close the system we need to specify the boundary and the initial conditions. We assume that the body is mechanically and energetically isolated and that there is no flux of chemical concentration through the boundary, i.e., we prescribe the following boundary conditions on $I \times \partial \Omega$

$$
\begin{aligned}
\boldsymbol{v} & =\mathbf{0}, \\
\mathbf{q}_{\mathfrak{c}}^{i} \cdot \boldsymbol{\nu} & =0 \\
\mathbf{q}_{e} \cdot \boldsymbol{\nu} & =0,
\end{aligned}
$$$$
\text { for } i=1, \ldots, L \text {, }
$$

where $\boldsymbol{\nu}$ denotes the unit outward normal vector at $I \times \partial \Omega$. Finally, we consider the following initial conditions

$$
\boldsymbol{v}(0)=\boldsymbol{v}_{0}, \quad \mathfrak{c}(0)=\mathfrak{c}_{0}, \quad e(0)=e_{0},
$$

that are supposed to be satisfied in $\Omega$.

2.1. Mechanical and thermodynamical consistency of the model. In this subsection we show the natural requirement on the constitutive relations that ensures the consistency of the considered model. We also recall that the density $\varrho$ is assumed to be identically equal to one. First natural assumption is that the mass is conserved and therefore it follows from (2.3) that necessarily

$$
\sum_{i=1}^{L} r_{i}=0
$$

Next, since $\mathbf{q}_{\mathbf{c}}^{i}=\left(\rho_{i} \boldsymbol{v}_{i}-\rho_{i} \boldsymbol{v}\right)$, it directly follows from (2.2) that

$$
\sum_{i=1}^{L} \mathbf{q}_{\mathfrak{c}}^{i}=\sum_{i=1}^{L} \rho_{i} \boldsymbol{v}_{i}-\rho_{i} \boldsymbol{v}=\mathbf{0},
$$

which represents the necessary structural assumption on the flux $\mathbf{q}_{\mathbf{c}}$.

Finally, we show which restrictions on the model parameters follows from the second law of thermodynamics. For this purpose we first derive ${ }^{2}$ the equation for internal energy. Taking the scalar product of (2.6) with $\boldsymbol{v}$, using (2.8) and (2.9), we can formally deduce the following identity for the kinetic energy

$$
\left(\frac{|\boldsymbol{v}|^{2}}{2}\right)_{, t}+\operatorname{div}\left(\boldsymbol{v}\left(\frac{|\boldsymbol{v}|^{2}}{2}+p\right)\right)-\operatorname{div}(\mathbf{S} \boldsymbol{v})+\mathbf{S} \cdot \nabla \boldsymbol{v}=0 .
$$

Thus, subtracting (2.16) from (2.7) we get the following identity for the internal energy

$$
e_{, t}+\operatorname{div}\left(e \boldsymbol{v}+\mathbf{q}_{e}\right)=\mathbf{S} \cdot \nabla \boldsymbol{v} \quad \text { in } Q .
$$

In what follows we consider that the thermodynamics of the model can be expressed by the primitive quantities $(\mathfrak{c}, e)$ and according to the second law of thermodynamics, we assume that

\footnotetext{
${ }^{2}$ Note that such approach can be justified only for sufficiently smooth solution. If the kinetic energy identity (2.16) is not valid, which is the case if we cannot test (2.6) by $\boldsymbol{v}$, the resulting equality (2.17) must be changed by the inequality. We refer to [2] and [3] for more details.
} 
there exists an entropy $s=s^{*}(e, \mathfrak{c})$, where $s^{*}: \mathbb{R}_{+} \times \mathbb{R}^{L} \rightarrow \mathbb{R}$ is a concave function. Moreover, we require the validity of the entropy inequality, i.e.,

$$
s_{, t}+\operatorname{div}\left(s \boldsymbol{v}+\mathbf{q}_{s}\right) \geq 0 \quad \text { in } Q,
$$

where $\mathbf{q}_{s}: Q \rightarrow \mathbb{R}^{d}$ is the entropy flux. In order to "recover" (2.18) from (2.4) and (2.17), we define the temperature $\theta:=\theta^{*}(e, \mathfrak{c})$ and the vector of re-scaled chemical potentials $\zeta:=\zeta^{*}(e, \mathfrak{c})$, where the functions $\theta^{*}$ and $\zeta^{*}$ are defined as

$$
\theta^{*}:=\frac{1}{\partial_{e} s^{*}}, \quad \zeta^{*}:=-\partial_{\mathfrak{c}} s^{*} .
$$

Then, multiplying the $i$-th equation in $(2.4)$ by $-\zeta^{i}$ summing the result over $i=1, \ldots, L$, and multiplying (2.17) by $\theta^{-1}$ and finally summing the resulting identities, using (2.19) and (2.8), we find that

$$
s_{, t}+\operatorname{div}\left(s \boldsymbol{v}+\frac{\mathbf{q}_{e}}{\theta}-\mathbf{q}_{\mathfrak{c}} \zeta\right)=\frac{\mathbf{S} \cdot \nabla \boldsymbol{v}}{\theta}+\left(\mathbf{q}_{e} \cdot \nabla \frac{1}{\theta}-\mathbf{q}_{\mathfrak{c}} \cdot \nabla \zeta\right)-\mathfrak{r} \cdot \zeta .
$$

Comparing (2.18) and (2.20) we see that the right hand side of (2.20) must be nonnegative. Thus, to simplify the situation we require in what follows that each term ${ }^{3}$ on the right hand side has a correct sign, i.e.,

$$
\begin{aligned}
\mathbf{S} \cdot \nabla \boldsymbol{v} & \geq 0, \\
\mathfrak{r} \cdot \zeta & \leq 0, \\
\mathbf{q}_{\mathfrak{c}} \cdot \nabla \zeta-\mathbf{q}_{e} \cdot \nabla \frac{1}{\theta} & \leq 0 .
\end{aligned}
$$

Note that (2.21)-(2.22) directly implies the restrictions on $\mathbf{S}$ and $\mathfrak{r}$ that will be even strengthen in the following subsection. We focus in the rest of this part on the restriction (2.23) and we still keep in mind that we want to model the cross effects caused by the temperature and the chemical potential gradients. Thus, for the flux $\mathbf{q}_{\mathfrak{c}}$ we consider the following generalization of the Fick law

$$
\mathbf{q}_{\mathfrak{c}}:=\mathbf{q}_{\zeta}(\mathfrak{c}, \theta, \nabla \zeta)+\overline{\mathbf{q}}_{\theta}(\mathfrak{c}, \theta, \nabla \theta)
$$

It means that while $\mathbf{q}_{\zeta}$ is driven mainly by the gradient of $\zeta$, the second part of the flux $\overline{\mathbf{q}}_{\theta}$ is driven mainly by the gradient of the temperature, i.e., describing the Soret effect. Similarly, we assume that the heat flux is also given by the sum representing two independent phenomena, i.e.,

$$
\mathbf{q}_{e}:=\mathbf{q}_{\theta}(\mathfrak{c}, \theta, \nabla \theta)+\overline{\mathbf{q}}_{\zeta}(\mathfrak{c}, \theta, \nabla \zeta)
$$

where again the first part models the heat flux caused by the temperature gradient, i.e., the Fourier law, and the second models the Dufour effect, i.e., flux driven by gradient of $\zeta$. Thus, substituting (2.24)-(2.25) into (2.23) and using the facts that $\mathbf{q}_{\zeta}$ is independent of the temperature gradient and similarly that $\mathbf{q}_{\theta}$ is independent of the gradient of $\zeta$ we see that it reduces to the following set of conditions

$$
\begin{array}{r}
\mathbf{q}_{\zeta} \cdot \nabla \zeta \leq 0, \\
-\mathbf{q}_{\theta} \cdot \nabla \frac{1}{\theta} \leq 0, \\
\overline{\mathbf{q}}_{\theta} \cdot \nabla \zeta-\overline{\mathbf{q}}_{\zeta} \cdot \nabla \frac{1}{\theta} \leq 0 .
\end{array}
$$

In addition, since due to the assumed structure of $\overline{\mathbf{q}}_{\theta}$ and $\overline{\mathbf{q}}_{\zeta}$, we immediately see from $(2.26)_{3}$ that necessarily

$$
\overline{\mathbf{q}}_{\theta} \cdot \nabla \zeta=\overline{\mathbf{q}}_{\zeta} \cdot \nabla \frac{1}{\theta}
$$

which represents the key restriction on the structure of fluxes in order to guarantee (2.18), i.e., the second law of thermodynamics.

\footnotetext{
${ }^{3}$ Note that it is in fact very natural if we assume that each term depends on a different quantity. On the other hand we could again split (2.23) onto two parts and require that $\mathbf{q}_{e} \cdot \nabla \frac{1}{\theta} \geq 0$ and $-\mathbf{q}_{\mathfrak{c}} \cdot \nabla \zeta \geq 0$. However, this would immediately lead to the fact that the Soret and the Dufour effects are forbidden in the model, which is not the case considered here.
} 
2.2. Assumptions on constitutive relations. Here, we describe the precise assumptions on the model. We would like to emphasize once again here, that we are not interested in developing the most general theory and the most general model. We only want to introduce a model that is able to capture the cross-effects and which is thermodynamically consistent. To simplify the notation, we first introduce the so-called Gibbs simplex as

$$
G=\left\{x \in \mathbb{R}^{L}: \sum_{i=1}^{L} x_{i}=1 ; x_{i} \geq 0 \text { for all } i=1, \ldots, L\right\} .
$$

Then we denote $\ell:=(1, \ldots, 1)$ and $G^{\prime}:=\ell^{\perp}$ and denote the orthogonal projection $\mathcal{P}_{\ell}$, that will be frequently used in what follows, as

$$
\mathcal{P}_{\ell}: \mathbb{R}^{L} \rightarrow G^{\prime}
$$

Note that

$$
\mathcal{P}_{\ell} x:=x-\frac{(x \cdot \ell)}{|\ell|^{2}} \ell .
$$

Then we specify the assumptions that will finally guarantee (2.21)-(2.23). The first assumption is related to the source term $\mathfrak{r}$.

(A1) We assume that $\mathfrak{r}=\mathfrak{r}^{*}(\mathfrak{c}, \theta, \zeta): \mathbb{R}^{L} \times \mathbb{R}_{+} \times \mathbb{R}^{L} \rightarrow \mathbb{R}^{L}$ is continuous and there exist $0<r_{1} \leq r_{2}<\infty$ and $q \in[1, \infty)$ such that for all $(\mathfrak{c}, \theta, \zeta) \in \mathbb{R}^{L} \times \mathbb{R}_{+} \times \mathbb{R}^{L}$ there holds

$$
\begin{aligned}
\mathcal{P}_{\ell} \mathfrak{r}^{*}(\mathfrak{c}, \theta, \zeta) & =\mathfrak{r}^{*}(\mathfrak{c}, \theta, \zeta), \\
\mathfrak{r}^{*}(\mathfrak{c}, \theta, \zeta) \cdot \zeta & \leq-r_{1}\left|\mathcal{P}_{\ell} \zeta\right|^{q} \\
|\mathfrak{r}(\mathfrak{c}, \theta, \zeta)| & \leq r_{2}(1+|\zeta|)^{q-1}
\end{aligned}
$$

Note here, that $(2.28)_{1}$ is just the condition (2.14) and $(2.28)_{2}$ is a stronger version of $(2.22)$. The reason why we assume such stronger assumption is that it will help us to establish the minimum principle for concentration $\mathfrak{c}$ which will follow from the assumptions on the entropy introduced below. Moreover, it follows from $(2.28)_{1}$ that such coercivity cannot be prescribed in term of $\zeta$ and therefore the term on the right hand side of $(2.28)_{2}$ depends only on $\mathcal{P}_{\ell} \zeta$. The last assumption in (2.28) is then the corresponding growth estimate, depending now on the whole $\zeta$ and not only on its projection $\mathcal{P}_{\ell} \zeta$.

Next, we introduce the assumption on the structure of the flux $\mathbf{q}_{\mathfrak{c}}$.

(A2) We assume that that the flux $\mathbf{q}_{\mathfrak{c}}$ is given as

$$
\mathbf{q}_{\mathfrak{c}}^{i}=\left(\mathbf{q}_{\mathfrak{c}}^{*}(\mathfrak{c}, \theta, \nabla \theta, \nabla \zeta)\right)^{i}:=-\sum_{j=1}^{L} M^{i j}(\mathfrak{c}, \theta) \nabla \zeta^{j}-\mathfrak{m}^{i}(\mathfrak{c}, \theta) \nabla \frac{1}{\theta},
$$

where $M: \mathbb{R}^{L} \times \mathbb{R}_{+} \rightarrow \mathbb{R}_{\text {sym }}^{L \times L}$ is continuous symmetric matrix-valued function and $\mathfrak{m}$ : $\mathbb{R}^{L} \times \mathbb{R}_{+} \rightarrow \mathbb{R}^{L}$ is continuous vector-valued function. Moreover, we require the following coercivity and growth assumptions: there exist $0<M_{1} \leq M_{2}<\infty$ such that for all $(\mathfrak{c}, \theta, \zeta) \in \mathbb{R}^{L} \times \mathbb{R}_{+} \times \mathbb{R}^{L}$ there holds

$$
\begin{aligned}
M_{1}\left|P_{\ell} \zeta\right|^{2} & \leq \sum_{i, j=1}^{L} M^{i j}(\mathfrak{c}, \theta) \zeta^{i} \zeta^{j}, \quad|M(\mathfrak{c}, \theta)| \leq M_{2}, \\
|\mathfrak{m}(\mathfrak{c}, \theta)| & \leq M_{2} \min (1, \theta) .
\end{aligned}
$$

In addition, the following compatibility conditions are assumed:

$$
\sum_{i=1}^{L} M^{i j}(\mathfrak{c}, \theta)=\sum_{i=1}^{L} \mathfrak{m}^{i}(\mathfrak{c}, \theta)=0 .
$$

Note here, that (2.31) is the necessary assumption for flux given by (2.29) in order to guarantee (2.15). Next, in (2.30), the "maximal" possible definiteness of the corresponding matrix is assumed. Moreover, it follows from (2.31) that

$$
\mathbf{q}_{\mathfrak{c}}^{*}(\mathfrak{c}, \theta, \nabla \theta, \nabla \zeta)=\mathbf{q}_{\mathfrak{c}}^{*}\left(\mathfrak{c}, \theta, \nabla \theta, \nabla\left(\mathcal{P}_{\ell} \zeta\right)\right) .
$$


Note here, that the setting (2.29) corresponds to the splitting (2.24), where we just set

$$
\mathbf{q}_{\zeta}^{i}:=-\sum_{j=1}^{L} M^{i j} \nabla \zeta^{j}, \quad \overline{\mathbf{q}}_{\theta}:=-\mathfrak{m}^{i} \nabla \frac{1}{\theta},
$$

and we also see that from (2.30) it directly follows $(2.26)_{1}$.

Concerning the heat flux $\mathbf{q}_{e}$ we consider the following generalization of the Fourier law:

(A3) We assume that $\mathbf{q}_{e}$ is given as

$$
\mathbf{q}_{e}=\mathbf{q}_{e}^{*}(\mathfrak{c}, \theta, \nabla \theta, \nabla \zeta):=-\kappa(\mathfrak{c}, \theta) \nabla \theta-\sum_{i=1}^{L} \mathfrak{m}^{i}(\mathfrak{c}, \theta) \nabla \zeta^{i},
$$

where $\kappa: \mathbb{R}^{L} \times \mathbb{R}_{+} \rightarrow \mathbb{R}_{+}$is continuous and there exist $0<\kappa_{1} \leq \kappa_{2}<\infty$ such that for all $(\mathfrak{c}, \theta) \in \mathbb{R}^{L} \times \mathbb{R}_{+}$there holds

$$
\kappa_{1} \leq \kappa(\mathfrak{c}, \theta) \leq \kappa_{2}
$$

Similarly as above, it directly follows from (2.33) and (2.31) that

$$
\mathbf{q}_{e}^{*}(\mathfrak{c}, \theta, \nabla \theta, \nabla \zeta)=\mathbf{q}_{e}^{*}\left(\mathfrak{c}, \theta, \nabla \theta, \nabla\left(\mathcal{P}_{\ell} \zeta\right)\right) .
$$

To compare such setting with the previous subsection, we recall (2.25) and by the use of (2.33), we see that

$$
\mathbf{q}_{\theta}:=-\kappa(\mathfrak{c}, \theta) \nabla \theta, \quad \overline{\mathbf{q}}_{\zeta}:=-\sum_{i=1}^{L} \mathfrak{m}^{i}(\mathfrak{c}, \theta) \nabla \zeta^{i} .
$$

Note that $(2.26)_{2}$ is then a direct consequence of $(2.34)$ and the identity $(2.27)$ then follows from $(2.33)$ and $(2.29)$.

Next, for the constitutively determined part of the Cauchy stress we assume the following:

(A4) We assume that $\mathbf{S}$ is given as

$$
\mathbf{S}:=\mathbf{S}^{*}(\mathfrak{c}, \theta, \mathbf{D}(\boldsymbol{v})),
$$

where $\mathbf{D}(\boldsymbol{v}):=\frac{1}{2}\left(\nabla \boldsymbol{v}+(\nabla \boldsymbol{v})^{T}\right)$ is the symmetric part of the velocity gradient. Moreover, we assume that $\mathbf{S}^{*}(\mathfrak{c}, \theta, \mathbf{D}): \mathbb{R}^{L} \times \mathbb{R}_{+} \times \mathbb{R}_{\text {sym }}^{d \times d} \rightarrow \mathbb{R}_{\text {sym }}^{d \times d}$ is continuous symmetric matricesvalued mapping and that there exist $q \in(1, \infty)$ and $0<\nu_{1} \leq \nu_{2}<\infty$ such that for all $(\mathfrak{c}, \theta) \in \mathbb{R}^{L} \times \mathbb{R}_{+}$and all $\mathbf{D}, \mathbf{B} \in \mathbb{R}_{\text {sym }}^{d \times d}$ there holds

$$
\begin{aligned}
& \mathbf{S}^{*}(\mathfrak{c}, \theta, \mathbf{0})=\mathbf{0}, \mathbf{S}^{*}(\mathfrak{c}, \theta, \mathbf{D}) \cdot \mathbf{D} \geq \nu_{1}|\mathbf{D}|^{r}-\nu_{2},\left|\mathbf{S}^{*}(\mathfrak{c}, \theta, \mathbf{D})\right| \leq \nu_{2}\left(1+|\mathbf{D}|^{r-1}\right), \\
& \left(\mathbf{S}^{*}(\mathfrak{c}, \theta, \mathbf{D})-\mathbf{S}^{*}(\mathfrak{c}, \theta, \mathbf{B})\right) \cdot(\mathbf{D}-\mathbf{B}) \geq 0 .
\end{aligned}
$$

Note that (2.36) is rather standard assumption on the Cauchy stress and represents its $r$-coercivity, $(r-1)$-growth and the monotonicity. Moreover, from (2.36) one can directly obtain (2.21) as a consequence of the monotonicity. Such model is capable to describe a lot of standard fluids. In fact we could even include a more general model based on the maximal monotone graph setting (suitable eg. for the Bingham fluid) but for simplicity we do not consider it here and refer the interested reader to $[4,5]$ for more details.

Finally we specify the assumptions on the entropy. The first and the key restriction is that the entropy is split:

(A5) The entropy $s=s^{*}(\mathfrak{c}, e)$ satisfies

$$
s=s^{*}(\mathfrak{c}, e):=s_{\mathfrak{c}}^{*}(\mathfrak{c})+s_{e}^{*}(e) .
$$

The assumption (2.37) plays the crucial role in the analysis, particularly in deriving a proper a priori estimates for the temperature and temperature gradient. On the other hand the following set of condition is quiet standard and ensures the nonnegativity of the temperature, gives the growth condition for the heat capacity and in particular enable us to prove that $\mathfrak{c} \in G$. We start with the assumptions on $s_{e}^{*}$. 
(A6) The function $s_{e}^{*}: \mathbb{R}_{+} \rightarrow \mathbb{R}$ is strictly increasing concave $\mathcal{C}^{2}$ function. Moreover, there exist positive constants $C_{1}$ and $C_{2}$ such that for all $e \in \mathbb{R}_{+}$there holds

$$
\begin{aligned}
s_{e}^{*}(e) & \leq C_{1} \ln e+C_{2}(e+1), \\
\frac{C_{1}}{e} & \leq \partial_{e} s_{e}^{*}(e) \leq \frac{C_{2}}{e}, \\
C_{1} & \leq-\frac{\partial_{e^{2}}^{2} s_{e}^{*}(e)}{\left(\partial_{e} s_{e}^{*}(e)\right)^{2}} \leq C_{2} .
\end{aligned}
$$

The temperature $\theta=\theta^{*}(e)$ is then defined as

$$
\theta^{*}:=\frac{1}{\partial_{e} s_{e}^{*}}
$$

and it directly follows from (2.39) that

$$
C_{2}^{-1} \theta^{*}(e) \leq e \leq C_{1}^{-1} \theta^{*}(e)
$$

Finally, we specify the assumptions on $s_{\mathfrak{c}}^{*}$.

(A7) The function $s_{\mathfrak{c}}^{*}:[0, \infty)^{L} \rightarrow \mathbb{R}$ is a concave continuous function such that $s^{*} \in \mathcal{C}^{2}\left((0, \infty)^{L}\right)$ and there exists a positive constant $C_{1}$ such that for all $\mathfrak{c}, x \in \mathbb{R}^{L}$ there holds

$$
C_{1}|x|^{2} \leq-\sum_{i, j=1}^{L} \partial_{c_{i} c_{j}}^{2} s_{\mathfrak{c}}^{*}(\mathfrak{c}) x_{i} x_{j} .
$$

In addition, we assume that for all $K>0$ there exists $\varepsilon>0$ such that

$$
\left|\partial_{\mathfrak{c}} s_{\mathfrak{c}}(\mathfrak{c})\right| \leq K \Longrightarrow c_{i} \geq \varepsilon \text { for all } i=1, \ldots, L .
$$

The vector of chemical potentials $\zeta=\zeta^{*}(\mathfrak{c})$ is then defined as

$$
\zeta^{*}:=-\partial_{\mathfrak{c}} s_{\mathfrak{c}}^{*}
$$

While the assumption (2.42) is rather standard, the implication (2.43) is required in the paper to provide that the concentrations are nonnegative. Indeed, since we do not assume any further structural properties of $\mathbf{q}_{\mathfrak{c}}^{*}$ and $\mathfrak{r}^{*}$, which would lead to the minimum principle for $\mathfrak{c}$ and which would lead to rather nonphysical setting, we combine $(2.28)_{2}$ with $(2.43)$. Then a uniform positivity for all component of $\mathfrak{c}$ follows from (2.43) provided we control $\zeta$. This will be however the consequence of the entropy inequality and the assumption $(2.28)_{2}$.

2.3. Examples for Cauchy stress, entropy and the diffusion matrix. Several typical examples of constitutive relations that satisfy (A1) $-(\mathbf{A} 7)$ is presented in this subsection. We do not discuss so much the most general possible setting for the Cauchy stress and our prototype model case that satisfies the required assumption is of the form

$$
\mathbf{S}^{*}(\mathfrak{c}, \theta, \mathbf{D}):=\nu_{0}(\mathfrak{c}, \theta)\left(1+|\mathbf{D}|^{2}\right)^{\frac{r-2}{2}} \mathbf{D},
$$

which is often called the Ladyzhenskaya model. For much better description and for more general setting we refer to [5].

Next, the prototype (frequently used) example of the entropy is the following

$$
s^{*}(\mathfrak{c}, e)=a \ln e-b \sum_{i=1}^{L} c_{i} \ln c_{i} \quad a, b>0
$$

and it is evident that the assumptions (A5)-(A7) are satisfied.

Concerning the diffusion matrices, we present here two examples. The first one is generated by the procedure introduced in [30] (see also the identical technique for less general setting in [13]). Thus, the first model example is given by the following multiplication

$$
M(\mathfrak{c}, \theta)=M_{2}^{T}(\mathfrak{c}, \theta) M_{1}(\mathfrak{c}, \theta) M_{2}(\mathfrak{c}, \theta),
$$


where $M_{1}$ and $M_{2}$ are defined as (we do not sum over repeating indices)

$$
\begin{aligned}
& M_{1}^{i j}=f_{i}(\mathfrak{c}, \theta) \delta_{i j}, \\
& M_{2}^{i j}=\delta^{i j}-\frac{f_{j}(\mathfrak{c}, \theta)}{\mathfrak{f}(\mathfrak{c}, \theta) \cdot \ell} .
\end{aligned}
$$

with $\mathfrak{f}:=\left(f_{1}, \ldots, f_{L}\right)$ being a continuous function such that $f_{i} \geq C_{1}>0$. It is easy to check that $M_{1}$ is positively definite and $\operatorname{ker} M_{2}=\operatorname{span}\{\ell\}$. The resulting matrix is

$$
M^{i j}=f_{i}(\mathfrak{c}, \theta)\left(\delta_{i j}-\frac{f_{j}(\mathfrak{c}, \theta)}{\mathfrak{f}(\mathfrak{c}, \theta) \cdot \ell}\right)
$$

and it is obvious that it fulfills (A2). The second example is then related to the case when we want the linear diffusion with no cross effects in $L-1$ components. For such cases, it seems to be appropriate to use the matrix of the following form

$$
M^{i j}(\mathfrak{c}, \theta):= \begin{cases}f_{j}(\mathfrak{c}, \theta) \delta_{i j} & \text { for } i<L \& j<L, \\ -f_{j}(\mathfrak{c}, \theta) & \text { for } i=L \& j<L, \\ -f_{i}(\mathfrak{c}, \theta) & \text { for } i<L \& j=L, \\ \mathfrak{f}(\mathfrak{c}, \theta) \cdot \ell-f_{L}(\mathfrak{c}, \theta) & \text { for } i=L \& j=L,\end{cases}
$$

which is useful mainly for dilute solutions. Again the functions $f_{i}$ are continuous and bounded from below. Moreover, it directly follows from the definition that (2.31) is satisfied. To check also (2.30), we first notice that for all $x \in \mathbb{R}^{L}$ such that $x_{L}=0$, we have

$$
M x \cdot x \geq C_{1}|x|^{2} .
$$

Consequently, we see that for arbitrary $x \in \mathbb{R}^{L}$

$$
\begin{aligned}
M x \cdot x & =M\left(x-x_{L} \ell\right) \cdot\left(x-x_{L} \ell\right)+x_{L}^{2} M \ell \cdot \ell-x_{L} M x \cdot \ell-x_{L} M \ell \cdot x \\
& =M\left(x-x_{L} \ell\right) \cdot\left(x-x_{L} \ell\right) \geq C_{1}\left|x-x_{L} \ell\right|^{2},
\end{aligned}
$$

where for the second equality we used (2.31) and for the final inequality we use (2.44). However, using the Young inequality, we see that

$$
\begin{aligned}
\left|x-x_{L} \ell\right|^{2} & =|x|^{2}+x_{L}^{2}|\ell|^{2}-2 x_{L} x \cdot \ell \geq|x|^{2}+x_{L}^{2}|\ell|^{2}-\frac{(x \cdot \ell)^{2}}{|\ell|^{2}}-x_{L}^{2}|\ell|^{2} \\
& =\left|\mathcal{P}_{\ell} x\right|^{2}
\end{aligned}
$$

and (2.30) follows.

\section{Function spaces, aUXiliary RESUlts AND DEFinition of a WEAK SOlution}

In this section we introduce all necessary notation, the definition of a weak solution to (2.4)(2.13) provided that the assumptions (A1) $-(\mathbf{A} 7)$ hold, and introduce the main theorems of the paper. Thus, we use the standard notation for the Lebesgue space of scalar-, vector- and tensorvalued functions, i.e., $L^{p}(\Omega), L^{p}\left(\Omega ; \mathbb{R}^{d}\right)$ and $L^{p}\left(\Omega ; \mathbb{R}^{d \times d}\right)$. In the similar way we introduce also the corresponding Sobolev spaces $W^{1, p}$ and the Sobolev spaces with zero trace $W_{0}^{1, p}$. Further, to simplify the notation, we set

$$
\begin{aligned}
W_{0, \text { div }}^{1, p} & :=\left\{\boldsymbol{v} \in W_{0}^{1, p}\left(\Omega ; \mathbb{R}^{d}\right): \operatorname{div} \boldsymbol{v}=0\right\}, \\
L_{0, \text { div }}^{2} & :=\overline{W_{0, \text { div }}^{1,2}\|\cdot\|_{2}} .
\end{aligned}
$$

Next, for $X$ being a Banach space, we denote $W^{k, p}(0, T ; X)$ the standard Sobolev-Bochner space. In addition, by $\mathcal{M}(0, T ; X)$ we always mean the space of $X$-valued measures. Further, to simplify the notation, for any $a \in L^{p}$ and $b \in L^{p^{\prime}}$ we set

$$
(a, b):=\int_{\Omega} a b d x
$$

and we use the same notation also for vector- or tensor-valued functions. Moreover, by the symbol $a \otimes b$ we will denote matrix with entries $(a \otimes b)_{i j}:=a_{i} b_{j}$. 
3.1. Definition of a weak solution and main results. Hence, having defined the proper function spaces, we can introduce the notion of a weak solution as follows.

Definition 3.1. Let $\Omega \subset \mathbb{R}^{d}$ be an open bounded Lipschitz domain and $d=2,3$. Assume that (A1)-(A7) are satisfied with $r \geq \frac{3 d+2}{d+2}$ and $q>2$. Moreover, assume that the initial data fulfill

$$
\begin{aligned}
\boldsymbol{v}_{0} & \in L_{0, \mathrm{div}}^{2}, \\
\mathfrak{c}_{0} & \in G \quad \text { a.e. in } \Omega, \\
e_{0} & \in L^{1}(\Omega), \\
s^{*}\left(\mathfrak{c}_{0}, e_{0}\right) & \in L^{1}(\Omega) .
\end{aligned}
$$

We say that $\left(\boldsymbol{v}, e, \mathfrak{c}, \mathbf{S}, \mathbf{q}_{\mathfrak{c}}, \mathbf{q}_{e}, s, \theta, \zeta, \mathfrak{r}\right)$ is a weak solution to (2.4)-(2.13) if

$$
\begin{aligned}
\boldsymbol{v} & \in L^{r}\left(0, T ; W_{0, \text { div }}^{1, r} \cap W^{1, r^{\prime}}\left(0, T ;\left(W_{0, \text { div }}^{1, r}\right),\right.\right. \\
\mathfrak{c} & \in L^{2}\left(0, T ; W^{1,2}\left(\Omega ; \mathbb{R}^{L}\right)\right), \quad \mathfrak{c} \in G \text { a. e. in } Q, \\
\mathfrak{c}_{, t} & \in\left(L^{2}\left(0, T ; W^{1,2}\left(\Omega ; \mathbb{R}^{L}\right)\right) \cap L^{q^{\prime}}\left(0, T ; L^{q^{\prime}}\left(\Omega ; \mathbb{R}^{L}\right)\right)\right)^{*}, \\
\mathbf{q}_{\mathfrak{c}} & \in L^{2}\left(0, T ; L^{2}\left(\Omega ; \mathbb{R}^{L \times d}\right)\right), \\
\mathbf{q}_{e} & \in L^{p}\left(0, T ; L^{p}\left(\Omega ; \mathbb{R}^{d}\right)\right) \quad \text { for all } 1 \leq p<\frac{d+2}{d+1}, \\
e & \in W^{1,1}\left(0, T ; W^{-1,1}(\Omega)\right), \\
e, \theta & \in L^{\infty}\left(0, T ; L^{1}(\Omega)\right) \cap L^{p}\left(0, T ; W^{1, p}(\Omega)\right) \\
\mathbf{S} & \in L^{r^{\prime}}\left(0, T ; L^{r^{\prime}}\left(\Omega ; \mathbb{R}_{s y m}^{d \times d}\right)\right), \\
\ln \theta & \in L^{\infty}\left(0, T ; L^{1}(\Omega)\right) \cap L^{2}\left(0, T ; W^{1,2}(\Omega)\right), \\
s, t & \in \mathcal{M}\left(0, T ; W^{-1,1}(\Omega)\right), \\
\zeta & \in L^{q}\left(0, T ; L^{q}\left(\Omega ; \mathbb{R}^{L}\right)\right), \\
\mathcal{P}_{\ell} \zeta & \in L^{2}\left(0, T ; W^{1,2}\left(\Omega ; \mathbb{R}^{L}\right)\right), \\
\mathfrak{r} & \in L^{q^{\prime}}\left(0, T ; L^{q^{\prime}}\left(\Omega ; \mathbb{R}^{L}\right)\right)
\end{aligned}
$$

and for almost all $t \in(0, T)$ the following equalities hold

$$
\begin{aligned}
\left\langle\boldsymbol{v}_{, t}, \boldsymbol{w}\right\rangle-(\boldsymbol{v} \otimes \boldsymbol{v}, \nabla \boldsymbol{w})+(\mathbf{S}, \nabla \boldsymbol{w}) & =0 & & \text { for all } \boldsymbol{w} \in W_{0, \mathrm{div}}^{1, \infty}, \\
\left\langle\mathfrak{c}_{, t}, \boldsymbol{u}\right\rangle-(\mathfrak{c} \otimes \boldsymbol{v}, \nabla \boldsymbol{u})-\left(\mathbf{q}_{\mathfrak{c}}, \nabla \boldsymbol{u}\right) & =(\mathfrak{r}, \boldsymbol{u}) & & \text { for all } \boldsymbol{u} \in W^{1, \infty}\left(\Omega ; \mathbb{R}^{L}\right), \\
\left\langle e_{, t}, u\right\rangle-(e \boldsymbol{v}, \nabla u)-\left(\mathbf{q}_{e}, \nabla u\right) & =(\mathbf{S} \cdot \nabla \boldsymbol{v}, u) & & \text { for all } u \in W^{1, \infty}(\Omega),
\end{aligned}
$$

and we also assume that the entropy inequality is satisfied in the following sense

$$
\langle s, t, u\rangle-\left(s \boldsymbol{v}+\frac{\mathbf{q}_{e}}{\theta}-\mathbf{q}_{\mathfrak{c}} \zeta, \nabla u\right) \geq\left(\frac{\mathbf{S} \cdot \nabla \boldsymbol{v}}{\theta}, u\right)+\left(\mathbf{q}_{e} \cdot \nabla \frac{1}{\theta}-\mathbf{q}_{\mathfrak{c}} \cdot \nabla \zeta, u\right)-(\mathfrak{r} \cdot \zeta, u)
$$

for all nonnegative $u \in W^{1 \infty}(\Omega)$. Moreover, we require that the following identities hold almost everywhere in $Q$

$$
\begin{aligned}
\mathbf{S} & =\mathbf{S}^{*}(\mathfrak{c}, \theta, \mathbf{D}(\boldsymbol{v})), \\
\mathbf{q}_{\mathfrak{c}} & =\mathbf{q}_{\mathfrak{c}}^{*}(\mathfrak{c}, \theta, \nabla \zeta, \nabla \theta), \\
\mathbf{q}_{e} & =\mathbf{q}_{e}^{*}(\mathfrak{c}, \theta, \nabla \zeta, \nabla \theta), \\
\mathfrak{r} & =\mathfrak{r}^{*}(\mathfrak{c}, \theta, \zeta), \\
\theta & =\theta^{*}(e), \\
s & =s_{e}^{*}(e)+s_{\mathfrak{c}}^{*}(\mathfrak{c}), \\
\zeta & =\zeta^{*}(\mathfrak{c}) .
\end{aligned}
$$


In addition, we assume that the initial data are attained in the following sense

$$
\lim _{t \rightarrow 0_{+}}\left\|\boldsymbol{v}(t)-\boldsymbol{v}_{0}\right\|_{2}+\left\|\mathfrak{c}(t)-\mathfrak{c}_{0}\right\|_{2}+\left\|e(t)-e_{0}\right\|_{1}=0 .
$$

Having a notion of a weak solution we formulate the main results of the paper. First, the easier one, is the weak stability result for any sequence of sufficiently smooth solution.

Theorem 3.1. Let $\Omega \subset \mathbb{R}^{d}$ be a Lipschitz domin with $d=2,3$ and $T>0$ be arbitrary. Assume that (A1)-(A7) are satisfied wit $r \geq \frac{3 d+2}{d+2}$ and $q>2$. Let $\left(\boldsymbol{v}^{n}, e^{n}, \mathfrak{c}^{n}, \mathbf{S}^{n}, \mathbf{q}_{\mathfrak{c}}^{n}, \mathbf{q}_{e}^{n}, \zeta^{n}, \theta^{n}, s^{n}, \mathfrak{r}^{n}\right)$ be a sequence of smooth weak solutions in the sense od Definition 3.1 corresponding to initial data $\left(\boldsymbol{v}_{0}^{n}, e_{0}^{n}, \mathfrak{c}_{0}^{n}\right)$ and let

$$
\lim _{n \rightarrow \infty}\left\|\boldsymbol{v}_{0}^{n}-\boldsymbol{v}_{0}\right\|_{2}+\left\|e_{0}^{n}-e_{0}\right\|_{1}+\left\|\mathfrak{c}_{0}^{n}-\mathfrak{c}_{0}\right\|_{2}+\left\|s^{*}\left(e_{0}^{n}, \mathfrak{c}_{0}^{n}\right)-s^{*}\left(e_{0}, \mathfrak{c}_{0}\right)\right\|_{1}=0
$$

Then we can extract a subsequence that we do not relabel and we can find $\left(\boldsymbol{v}, e, \mathfrak{c}, \mathbf{S}, \mathbf{q}_{\mathfrak{c}}, \mathbf{q}_{e}, \zeta, \theta, s, \mathfrak{r}\right)$ being a weak solution in the sense of Definition 3.1 with the initial data $\left(\boldsymbol{v}_{0}, e_{0}, \mathfrak{c}_{0}\right)$ such that

$$
\left(\boldsymbol{v}^{n}, e^{n}, \mathfrak{c}^{n}, \mathbf{S}^{n}, \mathbf{q}_{\mathfrak{c}}^{n}, \mathbf{q}_{e}^{n}, \zeta^{n}, \theta^{n}, s^{n}, \mathfrak{r}^{n}\right) \rightarrow^{*}\left(\boldsymbol{v}, e, \mathfrak{c}, \mathbf{S}, \mathbf{q}_{\mathfrak{c}}, \mathbf{q}_{e}, \zeta, \theta, s, \mathfrak{r}\right)
$$

in the weak* topology of the spaces introduced in Definition 3.1.

The proof of Theorem 3.1 is based on the entropy estimates and on monotone operator theory. In fact the only difficulty is to prove the attainment of the initial data. Although the relatively simple and standard proof, we present it here. The reason is twofold. First, we show that the the choice of the function spaces in Definition 3.1 is adequate and corresponds to the nature of the problem. Second, it is a preparation for the proof of the second result of the paper - the following existence theorem.

Theorem 3.2. Let $\Omega \subset \mathbb{R}^{d}$ be a Lipschitz domain with $d=2,3$ and $T>0$ be arbitrary. Assume that (A1)-(A7) are satisfied wit $r \geq \frac{3 d+2}{d+2}$ and $q>2$. Then for arbitrary initial data fulfilling (3.1)-(3.4) there exists a weak solution in the sense of Definition 3.1.

Also the proof of Theorem 3.2 is based on the entropy estimates and monotone operator theory. This is also the reason why we restrict ourselves onto the case $r \geq \frac{3 d+2}{d+2}$. Although it might seem to be a trivial task in view of Theorem 3.1, it is not the case. In fact, one has to find a proper approximative scheme that allows one to get a priori estimate coming from the entropy inequality. But since for such an estimate the very special choice of the test function is used, the usual Galerkin approximation and/or fixed point technique cannot be easily applied. Further, we would like to emphasize here, that according to our best knowledge, it is the first existence theorem, for a model capable to describe the Soret and the Dufour effect as well.

Moreover, the uniform estimates can be in principle obtained even for all $r \geq \frac{2 d}{d+2}$ and the only limitation in the proof of the main theorem is the passage to the limit in the term on the right hand side of the equation for the internal energy. However, following the ideas from [10], we can "redefine" the notion of a weak solution such that we replace (3.7) by the balance of the global energy (2.7) (which can be even integrated over $\Omega$ ) to get still consistent definition of a weak solution. (Here consistent means that if the solution in the sense of this new definition is smooth then it is also a solution to the original problem.) But for such refine notion of a weak solution, we can follow the method developed in [5] and [7] and to establish the existence of a weak solution for all $r>\frac{2 d}{d+2}$. But since, it is only the technical extension and the heart of the proof relies on getting a priori estimates, we do not present it here for simplicity.

3.2. Auxiliary results. In this part, we recall some know facts used later in the proofs of the main theorem. First, the following simple relation

$$
\mathcal{P}_{\ell}(\nabla \zeta)=\nabla\left(\mathcal{P}_{\ell} \zeta\right)
$$

valid for all $\mathbb{R}^{L}$-valued functions $\zeta$ will be used in what follows. We recall the Korn inequality.

Lemma 3.3 (Korn inequality (see [19])). Let $\Omega$ be an open bounded Lipschitz domain and let $1<p<\infty$ be arbitrary. Then there exists $C(p, \Omega)$ such that for all $\boldsymbol{v} \in W_{0}^{1, p}\left(\Omega ; \mathbb{R}^{d}\right)$ there holds

$$
\|\boldsymbol{v}\|_{1, r} \leq C(p, \Omega)\|\mathbf{D}(\boldsymbol{v})\|_{r} .
$$


We also recall the following interpolation inequality.

Lemma 3.4 (Interpolation (see e.g. [3])). Let $\Omega$ be an open bounded Lipschitz domain. Then for all $p \geq \frac{2 d}{d+2}$ there exists $C(p, \Omega)$ such that for all $u \in W^{1, p}(\Omega)$ there holds

$$
\|u\|_{\frac{(d+2) p}{d}}^{\frac{(d+2) p}{d}} \leq C\|u\|_{2}^{\frac{2 p}{d}}\|u\|_{1, p}^{p}
$$

We end this auxiliary part by formulation an algebraic lemma that will finally enable us to get proper estimates from the entropy inequality.

Lemma 3.5. Let $s_{\mathfrak{c}}^{*}$ satisfy $(\mathbf{A} 7)$ and let

$$
\zeta^{*}(\mathfrak{c}):=-\partial_{\mathfrak{c}} s_{\mathfrak{c}}^{*}(\mathfrak{c}) .
$$

Then there exists $C>0$ such that for all $\mathfrak{c} \in(0,1)^{L}$ there holds

$$
\left(\zeta^{*}(\mathfrak{c})\right)^{i} \leq C, \quad \text { and } \quad\left|\left(\zeta^{*}(\mathfrak{c})\right)^{i}\right| \leq \frac{C}{c_{i}} \quad \text { for all } i=1, \ldots, L .
$$

Moreover, there exists $C>0$ such that for all $\mathfrak{c} \in G$ we have

$$
\left|\zeta^{*}(\mathfrak{c})\right| \leq C\left(1+\left|\mathcal{P}_{\ell} \zeta^{*}(\mathfrak{c})\right|\right)
$$

Proof. First for any $0 \leq a \leq 2$, and any $\mathfrak{c} \in(0,2)^{L}$, we set

$$
\mathfrak{c}_{a}:=\left(a, c_{2}, \ldots, c_{L}\right)
$$

Then for any $0 \leq a \leq b \leq 2$, we can use the concavity of $s_{\mathfrak{c}}^{*}$ (which implies that $\zeta^{1}\left(\mathfrak{c}_{t}\right)$ is nondecreasing function of $t$ ) to deduce that

$$
\left(\zeta^{*}\left(\mathfrak{c}_{a}\right)\right)^{1} \leq \frac{s_{\mathfrak{c}}^{*}\left(\mathfrak{c}_{a}\right)-s_{\mathfrak{c}}^{*}\left(\mathfrak{c}_{b}\right)}{b-a} \leq\left(\zeta^{*}\left(\mathfrak{c}_{b}\right)\right)^{1} .
$$

Thus, setting $a=c_{1}$ and $b=2$ we have for all $\mathfrak{c} \in(0,1)^{L}$ that

$$
\left(\zeta^{*}(\mathfrak{c})\right)^{1} \leq \frac{s_{\mathfrak{c}}^{*}(\mathfrak{c})-s_{\mathfrak{c}}^{*}\left(\mathfrak{c}_{2}\right)}{2-c_{1}} \leq C .
$$

Similarly, setting $b:=c_{1}$ and $a=0$ we get

$$
\left(\zeta^{*}(\mathfrak{c})\right)^{1} \geq \frac{s_{\mathfrak{c}}^{*}\left(\mathfrak{c}_{0}\right)-s_{\mathfrak{c}}^{*}(\mathfrak{c})}{c_{1}} \geq-\frac{C}{c_{1}}
$$

from which the estimate (3.20) directly follows. To prove (3.21), we first note that for any $\mathfrak{c} \in G$ there exists $i$ such that $1 \geq c_{i} \geq \frac{1}{L}$. For simplicity, assume that $i=L$. Then, it follows from (3.20) that $\left|\zeta^{* L}\right| \leq C L$. Consequently, using the Young inequality, we get

$$
\begin{aligned}
\left|\zeta^{*}(\mathfrak{c})\right|^{2} & =\left|\mathcal{P}_{\ell} \zeta^{*}(\mathfrak{c})\right|^{2}+\frac{1}{L}\left(\zeta^{*}(\mathfrak{c}) \cdot \ell\right)^{2} \\
& =\left|\mathcal{P}_{\ell} \zeta^{*}(\mathfrak{c})\right|^{2}+\frac{1}{L}\left(\sum_{i=1}^{L-1}\left(\zeta^{*}(\mathfrak{c})\right)^{i}\right)^{2}+\frac{2}{L}\left(\zeta^{*}(\mathfrak{c})\right)^{L} \sum_{i=1}^{L-1}\left(\zeta^{*}(\mathfrak{c})\right)^{i}+\frac{1}{L}\left(\left(\zeta^{*}(\mathfrak{c})\right)^{L}\right)^{2} \\
& \leq\left|\mathcal{P}_{\ell} \zeta^{*}(\mathfrak{c})\right|^{2}+\frac{L-1}{L} \sum_{i=1}^{L-1}\left(\left(\zeta^{*}(\mathfrak{c})\right)^{i}\right)^{2}+\frac{2}{L}\left(\zeta^{*}(\mathfrak{c})\right)^{L} \sum_{i=1}^{L-1}\left(\zeta^{*}(\mathfrak{c})\right)^{i}+\frac{1}{L}\left(\left(\zeta^{*}(\mathfrak{c})\right)^{L}\right)^{2} \\
& \leq\left|\mathcal{P}_{\ell} \zeta^{*}(\mathfrak{c})\right|^{2}+\frac{L-\frac{1}{2}}{L}\left|\zeta^{*}(\mathfrak{c})\right|^{2}+C(L)\left(\left(\zeta^{*}(\mathfrak{c})\right)^{L}\right)^{2} \leq\left|\mathcal{P}_{\ell} \zeta^{*}(\mathfrak{c})\right|^{2}+\frac{L-\frac{1}{2}}{L}\left|\zeta^{*}(\mathfrak{c})\right|^{2}+C(L)
\end{aligned}
$$

Hence, moving the second term on the right hand side onto the left hand side, it is not difficult to observe the validity of (3.21). 


\section{Proof of Theorem 3.1}

We start with the proof of a priori estimates that also justify the choice of the function spaces specified in Definition 3.1. At this point we would like to refer to $[2,3]$ for more details concerning the estimated for the heat equation with a priori $L^{1}$ right hand side. First, we denote $(3.5)_{n}-(3.8)_{n}$ the identities valid for the $n$-th weak solution. Thus, setting $\boldsymbol{w}:=\boldsymbol{v}^{n}$ in $(3.5)_{n}$ and integrating with respect to time over $(0, t)$, we deduce (the convective terms vanishes) the energy identity

$$
\left\|\boldsymbol{v}^{n}(t)\right\|_{2}^{2}+2 \int_{0}^{t}\left(\mathbf{S}^{n}, \mathbf{D}\left(\boldsymbol{v}^{n}\right)\right) d \tau=\left\|\boldsymbol{v}_{0}^{n}\right\|_{2}^{2}
$$

Hence using (2.36), the Korn inequality, the interpolation inequality (3.19) and (3.17), we obtain

$$
\sup _{t \in(0, T)}\left\|\boldsymbol{v}^{n}(t)\right\|_{2}^{2}+\int_{0}^{T}\|\mathbf{S}\|_{r^{\prime}}^{r^{\prime}}+\left\|\boldsymbol{v}^{n}\right\|_{1, r}^{r}+\left\|\boldsymbol{v}^{n}\right\|_{\frac{(d+2) r}{d}}^{\frac{(d+2) r}{d}} d t \leq C
$$

and consequently from $(3.5)_{n}$ it follows (here we use the fact that $r>\frac{3 d+2}{d+2}$ ) that

$$
\int_{0}^{T}\left\|\boldsymbol{v}_{, t}^{n}\right\|_{\left(W_{0, \mathrm{div}}^{1, r}\right)^{*}}^{r^{\prime}} d t \leq C
$$

Next, we set $\boldsymbol{u}:=\ell \varphi$ in $(3.6)_{n}$, where $\varphi \in W^{1, \infty}$ is arbitrary, and by using (A1) and (A2), we deduce that

$$
\left\langle\left(\mathfrak{c}^{n} \cdot \ell\right)_{, t}, \varphi\right\rangle-\left(\left(\mathfrak{c}^{n} \cdot \ell\right) \boldsymbol{v}^{n}, \nabla \varphi\right)=0,
$$

which is nothing else than the transport equation for the quantity $\mathfrak{c}^{n} \cdot \ell$. Bust since $\mathfrak{c}_{0}^{n} \cdot \ell \equiv 1$ in $\Omega$ we see that

$$
\mathfrak{c}^{n} \cdot \ell \equiv 1 \quad \text { a.e. in } Q .
$$

Moreover, since $\zeta^{n}$ are assumed to be smooth we can use (2.43) to observe

$$
c_{i}^{n} \geq 0 \quad \text { a.e. in } Q \text { for all } i=1, \ldots, L .
$$

Consequently, we get

$$
\mathfrak{c}^{n} \in G \quad \text { a. e. in } Q .
$$

Similarly, setting $u \equiv 1$ in $(3.7)_{n}$ and integrating the result with respect to time over $(0, t)$, we get

$$
\int_{\Omega} e^{n}(t) d x=\int_{0}^{t} \int_{\Omega} \mathbf{S}^{n} \cdot \mathbf{D}\left(\boldsymbol{v}^{n}\right) d x d \tau+\int_{\Omega} e_{0}^{n} d x
$$

Since the entropies are assumed to be smooth, we see from (2.39) that $e \geq 0$ and therefore using (3.17), (2.36) and (4.2)

$$
\sup _{t \in(0, T)}\left\|e^{n}(t)\right\|_{1} \leq C .
$$

Finally, setting $u \equiv 1$ in $(3.8)_{n}$ and integrating the result with respect to time over $(0, t)$ we have

$$
\int_{\Omega} s^{n}(t)-s_{0}^{n} d x \geq \int_{0}^{t} \int_{\Omega} \frac{\mathbf{S}^{n} \cdot \nabla \boldsymbol{v}^{n}}{\theta^{n}}+\mathbf{q}_{e}^{n} \cdot \nabla \frac{1}{\theta^{n}}-\mathbf{q}_{\mathbf{c}}^{n} \cdot \nabla \zeta^{n}-\mathfrak{r}^{n} \cdot \zeta^{n} d x d \tau .
$$

First, since $e^{n}$ are nonnegative it follows from (2.39) that also $\theta^{n}$ are nonnegative. Consequently, using (2.36) we see that also the first term on the right hand side is nonnegative. Moreover, using (2.29) and (2.33) and the assumptions on the structure $\mathbf{q}_{e}^{*}$ and $\mathbf{q}_{\mathbf{c}}^{*}$ (see assumptions (A2)-(A3)), we deduce from (4.7) that

$$
\int_{\Omega} s^{n}(t)-s_{0}^{n} d x \geq \int_{0}^{t} \int_{\Omega} \frac{\kappa\left(\mathfrak{c}^{n}, \theta^{n}\right)\left|\nabla \theta^{n}\right|^{2}}{\left(\theta^{n}\right)^{2}}+M\left(\mathfrak{c}^{n}, \theta^{n}\right) \nabla \zeta^{n} \cdot \nabla \zeta^{n}-\mathfrak{r}^{n} \cdot \zeta^{n} d x d \tau .
$$

Thus, using (3.17), (4.6), (2.38), (2.28), (2.34) and (2.30) (note here, that we use the fact that $\mathcal{P}_{\ell}$ and $\nabla$ commute) we obtain

$$
\sup _{t \in(0, T)}\left\|\ln e^{n}(t)\right\|_{1}+\int_{0}^{T}\left\|\nabla \ln \theta^{n}\right\|_{2}^{2}+\left\|\nabla \mathcal{P}_{\ell} \zeta^{n}\right\|_{2}^{2}+\left\|\mathcal{P}_{\ell} \zeta^{n}\right\|_{q}^{q} d t \leq C .
$$


In addition, using (3.21), we deduce from (4.5) and (4.9) that

$$
\int_{0}^{T}\left\|\zeta^{n}\right\|_{q}^{q} d t \leq C .
$$

Consequently, it follows from (2.28), (2.30), (2.31), (4.9) and (4.10) that ${ }^{4}$

$$
\int_{0}^{T}\left\|\mathfrak{r}^{n}\right\|_{q^{\prime}}^{q^{\prime}}+\left\|\mathbf{q}_{\mathfrak{c}}^{n}\right\|_{2}^{2} d t \leq C .
$$

Thus, using (4.2), (4.11) we can deduce from $(3.6)_{n}$ that

$$
\left\|\mathfrak{c}_{, t}^{n}\right\|_{\left(L^{q}\left(0, T ; L^{q}\left(\Omega ; \mathbb{R}^{L}\right)\right) \cap L^{2}\left(0, T ; W^{1,2}\left(\Omega ; \mathbb{R}^{L}\right)\right)\right)^{*}} \leq C .
$$

Finally, using (2.42) and (4.9), we can also derive the uniform bound on $\nabla \mathfrak{c}^{n}$. Indeed, it follows from (2.42) that

$$
C_{1}\left|\nabla \mathfrak{c}^{n}\right|^{2} \leq \nabla \zeta^{n} \cdot \nabla \mathfrak{c}^{n}=\nabla \mathcal{P}_{\ell} \zeta^{n} \cdot \nabla \mathfrak{c}^{n}+\frac{\nabla\left(\zeta^{n} \cdot \ell\right) \cdot \nabla\left(\mathfrak{c}^{n} \cdot \ell\right)}{|\ell|^{2}}=\nabla \mathcal{P}_{\ell} \zeta^{n} \cdot \nabla \mathfrak{c}^{n},
$$

where the last identity follows from (4.5). Thus, integrating the result over $Q$, using the Young inequality and the a priori estimate (4.9) we obtain

$$
\int_{0}^{T}\left\|\mathfrak{c}^{n}\right\|_{1,2}^{2} d t \leq C
$$

Next, we focus on further estimates on the internal energy $e^{n}$ and consequently on the temperature $\theta^{n}$. We basically follow the standard procedure for the heat equation with $L^{1}$-right hand side (see eg. [3] for details), which is possible since we already control the term involving $\nabla \mathcal{P}_{\ell} \zeta^{n}$ in $\mathbf{q}_{e}$. Thus, setting $u:=\left(1+e^{n}\right)^{-\lambda}$ with arbitrary $\lambda \in(0,1)$ in $(3.7)_{n}$, we get (using the fact the the convective term vanishes and also the fact that the term on the right hand side is nonnegative)

$$
\frac{d}{d t} \int_{\Omega}\left(1+e^{n}\right)^{1-\lambda} d x \geq-\lambda(1-\lambda) \int_{\Omega} \frac{\mathbf{q}_{e}^{n} \cdot \nabla e^{n}}{\left(1+e^{n}\right)^{1+\lambda}} d x .
$$

Thus, integrating this with respect to time over $(0, T)$, using (4.6), (2.33) and (2.31), we obtain

$$
\int_{0}^{T} \int_{\Omega} \frac{\kappa\left(\mathfrak{c}^{n}, \theta^{n}\right) \nabla \theta^{n} \cdot \nabla e^{n}}{\left(1+e^{n}\right)^{1+\lambda}} d x d t \leq C(\lambda)+C(\lambda) \int_{0}^{T} \int_{\Omega}\left|\nabla \mathcal{P}_{\ell} \zeta^{n}\right|\left|\nabla e^{n}\right|\left(1+e^{n}\right)^{-1-\lambda} d x d t .
$$

Hence, using (2.34) and (2.39) to get lower bound for the term on the left hand side, and using the Young inequality to move the corresponding term from the right hand side, we deduce

$$
\int_{0}^{T} \int_{\Omega} \frac{\left|\nabla e^{n}\right|^{2}}{\left(1+e^{n}\right)^{1+\lambda}} d x d t \leq C(\lambda)+C(\lambda) \int_{0}^{T}\left\|\nabla \mathcal{P}_{\ell} \zeta^{n}\right\|_{2}^{2} d t \leq C(\lambda)
$$

where the last inequality follows from the a priori bound (4.9). Consequently, using (4.6) and (4.14) we get that

$$
\sup _{t \in(0, T)}\left\|\left(1+e^{n}\right)^{\frac{1-\lambda}{2}}\right\|_{2}+\int_{0}^{T}\left\|\left(1+e^{n}\right)^{\frac{1-\lambda}{2}}\right\|_{1,2}^{2} d t \leq C(\lambda) .
$$

Consequently, from the interpolation inequality (3.19) it follows that

$$
\int_{0}^{T}\left\|e^{n}\right\|_{\frac{d+2}{d}-\varepsilon}^{\frac{d+2}{d}-\varepsilon} d t \leq C\left(\varepsilon^{-1}\right) \quad \text { for all } \varepsilon>0
$$

\footnotetext{
${ }^{4}$ Here we heavily use the assumption (2.30), from which it follows that

$$
\left|\mathbf{q}_{\mathfrak{c}}\right| \leq C\left(\left|\nabla \mathcal{P}_{\ell} \zeta^{n}\right|+\left|\nabla \ln \theta^{n}\right|\right) .
$$
}


Going back to the estimates for the gradient of $e^{n}$, we use the Hölder inequality to conclude

$$
\begin{aligned}
\int_{0}^{T} & \left\|\nabla e^{n}\right\|_{\frac{d+2-\varepsilon}{d+1}}^{\frac{d+2-\varepsilon}{d+1}} d t=\int_{0}^{T} \int_{\Omega}\left(\frac{\left|\nabla e^{n}\right|^{2}}{\left(1+e^{n}\right)^{1+\lambda}}\right)^{\frac{d+2-\varepsilon}{2(d+1)}}\left(1+e^{n}\right)^{(1+\lambda)\left(\frac{d+2-\varepsilon}{2(d+1)}\right)} d x d t \\
& \leq\left(\int_{0}^{T} \int_{\Omega} \frac{\left|\nabla e^{n}\right|^{2}}{\left(1+e^{n}\right)^{1+\lambda}} d x d t\right)^{\frac{d+2-\varepsilon}{2(d+1)}}\left(\int_{0}^{T} \int_{\Omega}\left(1+e^{n}\right)^{(1+\lambda)\left(\frac{d+2-\varepsilon}{d+\varepsilon}\right)} d x d t\right)^{\frac{d+\varepsilon}{2(d+1)}} .
\end{aligned}
$$

Thus, using (4.14) with $\lambda=\varepsilon / d$ and (4.15), we see that

$$
\int_{0}^{T}\left\|\nabla e^{n}\right\|_{\frac{d+2-\varepsilon}{d+1}}^{\frac{d+2-\varepsilon}{d+1}} d t \leq C\left(\varepsilon^{-1}\right) \quad \text { for all } \varepsilon>0
$$

and consequently in view of $(2.40)$ we have

$$
\int_{0}^{T}\left\|\nabla \theta^{n}\right\|_{\frac{d+2-\varepsilon}{d+1}}^{\frac{d+2-\varepsilon}{d+1}} d t \leq C\left(\varepsilon^{-1}\right) \quad \text { for all } \varepsilon>0 .
$$

Since it follows from (2.31), (2.33) and (2.34) that

$$
\left|\mathbf{q}_{e}^{n}\right| \leq C\left(\left|\nabla \theta^{n}\right|+\min \left(1, \theta^{n}\right)\left|\nabla\left(\mathcal{P}_{\ell} \zeta^{n}\right)\right|\right),
$$

it is evident that by using (4.9) and (4.17) we can deduce that

$$
\int_{0}^{T}\left\|\frac{\mathbf{q}_{e}^{n}}{\theta^{n}}\right\|_{2}^{2}+\left\|\mathbf{q}_{e}^{n}\right\|_{\frac{d+2}{d+1}-\varepsilon}^{\frac{d+2}{d+1}-\varepsilon} d t \leq C\left(\varepsilon^{-1}\right) \quad \text { for all } \varepsilon>0 .
$$

Finally, having all above estimates it follows from $(3.7)_{n}$ that (note here that $d=2,3$ in order to bound the convective term)

$$
\int_{0}^{T}\left\|e_{, t}^{n}\right\|_{\left(W^{2, d}(\Omega)\right)^{*}} \leq C .
$$

Thus, having (4.2), (4.3), (4.5), (4.6), (4.9)-(4.13), (4.15), (4.16)-(4.19) we can extract a subsequence that we do not relabel such that

$$
\begin{array}{cl}
\boldsymbol{v}^{n} \rightarrow \boldsymbol{v} & \text { weakly in } W^{1, r^{\prime}}\left(0, T ;\left(W_{0, \text { div }}^{1, r}\right)^{*}\right) \cap L^{r}\left(0, T ; W_{0, \text { div }}^{1, r}\right), \\
\mathfrak{c}^{n} \boldsymbol{*}^{*} \mathfrak{c} & \text { weakly* in } L^{2}\left(0, T ; W^{1,2}\left(\Omega ; \mathbb{R}^{L}\right)\right) \cap L^{\infty}\left(0, T ; L^{\infty}\left(\Omega ; \mathbb{R}^{L}\right)\right), \\
\mathfrak{c}_{, t}^{n} \rightarrow \mathfrak{c}_{, t} & \text { weakly in }\left(L^{2}\left(0, T ; W^{1,2}\left(\Omega ; \mathbb{R}^{L}\right)\right) \cap L^{q}\left(0, T ; L^{q}\left(\Omega ; \mathbb{R}^{L}\right)\right)\right)^{*}, \\
e^{n} \rightarrow e & \text { weakly in } L^{s}\left(0, T ; W^{1, s}(\Omega)\right) \quad \text { for all } s \in\left[1, \frac{d+2}{d+1}\right), \\
e_{, t}^{n} \rightarrow^{*} e_{, t} & \text { weakly* in } \mathcal{M}\left(0, T ;\left(W^{2, d}(\Omega)\right)^{*}\right), \\
\theta^{n} \rightarrow \theta & \text { weakly in } L^{s}\left(0, T ; W^{1, s}(\Omega)\right) \quad \text { for all } s \in\left[1, \frac{d+2}{d+1}\right), \\
\mathbf{q}_{e}^{n} \rightarrow \mathbf{q}_{e} & \text { weakly in } L^{s}\left(0, T ; L^{s}\left(\Omega ; \mathbb{R}^{d}\right)\right) \quad \text { for all } s \in\left[1, \frac{d+2}{d+1}\right), \\
\mathbf{q}_{\mathfrak{c}}^{n} \rightarrow \mathbf{q}_{\mathfrak{c}} & \text { weakly in } L^{2}\left(0, T ; L^{2}\left(\Omega ; \mathbb{R}^{d \times L}\right)\right), \\
\mathbf{S}^{n} \rightarrow \mathbf{S} & \text { weakly in } L^{r^{\prime}}\left(0, T ; L^{r^{\prime}}\left(\Omega ; \mathbb{R}^{d \times d}\right)\right), \\
\zeta^{n} \rightarrow \zeta & \text { weakly in } L^{q}\left(0, T ; L^{q}\left(\Omega ; \mathbb{R}^{L}\right)\right), \\
\mathfrak{r}^{n} \rightarrow \mathfrak{r} & \text { weakly in } L^{q^{\prime}}\left(0, T ; L^{q^{\prime}}\left(\Omega ; \mathbb{R}^{L}\right)\right), \\
\mathcal{P}_{\ell} \zeta^{n} \rightarrow \mathcal{P}_{\ell} \zeta & \text { weakly in } L^{2}\left(0, T ; W^{1,2}\left(\Omega ; \mathbb{R}^{L}\right)\right) .
\end{array}
$$


Moreover, using the Aubin-Lions lemma, (4.9) and (4.18), we can strengthen the above convergence results to obtain

$$
\begin{aligned}
\boldsymbol{v}^{n} & \rightarrow \boldsymbol{v} & & \text { strongly in } L^{s}\left(0, T ; L^{s}\left(\Omega ; \mathbb{R}^{d}\right)\right) \quad \text { for all } s \in\left[1, \frac{(d+2) r}{d}\right), \\
\mathfrak{c}^{n} & \rightarrow \mathfrak{c} & & \text { strongly in } L^{s}\left(0, T ; L^{s}\left(\Omega ; \mathbb{R}^{L}\right)\right) \quad \text { for all } s \in[1, \infty) \\
e^{n} & \rightarrow e & & \text { strongly in } L^{s}\left(0, T ; L^{s}(\Omega)\right) \quad \text { for all } s \in\left[1, \frac{d+2}{d}\right), \\
\theta^{n} & \rightarrow \theta & & \text { strongly in } L^{s}\left(0, T ; L^{s}(\Omega)\right) \quad \text { for all } s \in\left[1, \frac{d+2}{d}\right), \\
\ln \theta^{n} & \rightarrow \ln \theta & & \text { weakly in } L^{2}\left(0, T ; L^{2}(\Omega)\right), \\
\frac{\mathbf{q}_{e}^{n}}{\theta^{n}} & -\frac{\mathbf{q}_{e}}{\theta} & & \text { weakly in } L^{2}\left(0, T ; L^{2}\left(\Omega, \mathbb{R}^{d}\right)\right) .
\end{aligned}
$$

All above convergence results allows us to conclude that $\left(\boldsymbol{v}, \mathfrak{c}, e, \theta, \mathbf{S}, \mathbf{q}_{e}, \mathbf{q}_{\mathbf{c}}, \mathfrak{r}\right)$ satisfies (3.5)(3.6). Moreover, using the Fatou lemma we can also conclude from (4.6), (4.9) and (4.34) that

$$
\sup _{t \in(0, T)}\|e(t)\|_{1}+\|\ln e(t)\|_{1} \leq C .
$$

In addition, if we are able to identify the weak limit of the term $\mathbf{S}^{n} \cdot \mathbf{D}\left(\boldsymbol{v}^{n}\right)$ we can obtain (3.7). Thus, it remains to check the validity of the entropy inequality (3.8), the attainment of the initial data (3.16) and the constitutive equations (3.9)-(3.15) together with the identification of the limit of the sequence $\mathbf{S}^{n} \cdot \mathbf{D}\left(\boldsymbol{v}^{n}\right)$.

First, the attainment of $\boldsymbol{v}_{0}$ and $\mathfrak{c}_{0}$ is standard for parabolic like equations and we refer e.g. to [19] for detailed proof. Then we can easily identify $\mathbf{S}$ by using the monotone operator theory. Indeed, setting $\boldsymbol{w}:=\boldsymbol{v}$ in (3.5) and integrating the result over $(0, T)$ we get the energy identity

$$
\|\boldsymbol{v}(T)\|_{2}^{2}+2 \int_{0}^{T}(\mathbf{S}, \mathbf{D}(\boldsymbol{v})) d t=\left\|\boldsymbol{v}_{0}\right\|_{2}^{2} .
$$

Next, letting $n \rightarrow \infty$ in (4.1), using weak lower semi-continuity and (3.17), we can observe that

$$
\limsup _{n \rightarrow \infty} \int_{0}^{T}\left(\mathbf{S}^{n}, \mathbf{D}\left(\boldsymbol{v}^{n}\right)\right) d t \leq \int_{0}^{T}(\mathbf{S}, \mathbf{D}(\boldsymbol{v})) d t .
$$

Due to the growth assumptions on $\mathbf{S}^{*}(2.36)$ and the convergence results (4.33) and (4.35), we can use the Lebesgue dominated convergence theorem, to conclude that for any symmetric-matrices valued $\mathbf{A} \in L^{r}\left(0, T ; L^{r}\left(\Omega ; \mathbb{R}^{d \times d}\right)\right)$

$$
\mathbf{S}^{*}\left(\mathfrak{c}^{n}, \theta^{n}, \mathbf{A}\right) \rightarrow \mathbf{S}^{*}(\mathfrak{c}, \theta, \mathbf{A}) \quad \text { strongly in } L^{r^{\prime}}\left(0, T ; L^{r^{\prime}}\left(\Omega ; \mathbb{R}^{d \times d}\right)\right) .
$$

Moreover, from the monotonicity of $\mathbf{S}^{*}$, see (2.36), we also have

$$
\int_{0}^{T}\left(\mathbf{S}^{n}-\mathbf{S}^{*}\left(\mathfrak{c}^{n}, \theta^{n}, \mathbf{A}\right), \mathbf{D}\left(\boldsymbol{v}^{n}\right)-\mathbf{A}\right) d t \geq 0 .
$$

Hence, letting $n \rightarrow \infty$ in (4.42), using (4.40), (4.41) and (4.28), we get

$$
\int_{0}^{T}\left(\mathbf{S}-\mathbf{S}^{*}(\mathfrak{c}, \theta, \mathbf{A}), \mathbf{D}(\boldsymbol{v})-\mathbf{A}\right) d t \geq 0 \quad \text { for all } \mathbf{A} \in L^{r}\left(0, T ; L^{r}\left(\Omega ; \mathbb{R}^{d \times d}\right)\right)
$$

and with the help of the Minty method we can deduce (3.9). Moreover, setting $\mathbf{A}:=\mathbf{D}(\boldsymbol{v})$ in (4.42), using (3.9) and (4.40) we obtain

$$
\left(\mathbf{S}^{n}-\mathbf{S}^{*}\left(\mathfrak{c}^{n}, \theta^{n}, \mathbf{D}(\boldsymbol{v})\right)\right) \cdot\left(\mathbf{D}\left(\boldsymbol{v}^{n}\right)-\mathbf{D}(\boldsymbol{v})\right) \rightarrow 0 \quad \text { strongly in } L^{1}\left(0, T ; L^{1}(\Omega)\right) .
$$

However, from this it directly follows (using (4.41) again) that

$$
\mathbf{S}^{n} \cdot \mathbf{D}\left(\boldsymbol{v}^{n}\right) \rightarrow \mathbf{S} \cdot \mathbf{D}(\boldsymbol{v}) \quad \text { weakly in } L^{1}\left(0, T ; L^{1}(\Omega)\right)
$$


and we can thus identify the term on the right hand side of (3.7). In addition, due to the weak convergence (4.45) (and consequently the uniform equi-integrability of the corresponding term) we can strengthen the convergence result (4.24) in the following way

$$
e_{, t}^{n} \rightarrow e_{, t} \quad \text { weakly in } L^{1}\left(0, T ;\left(W^{2, d}(\Omega)\right)^{*}\right) .
$$

Next, due to the continuity of $s^{*}, \zeta^{*}, \theta^{*}, \mathfrak{r}^{*}$ and the convergence results (4.33), (4.34), (4.29) and (4.30), it is easy to observe that (3.12)-(3.15) hold. To get validity also for (3.10)-(3.11), we use (2.32) and (2.35) and using the linearity of $\mathbf{q}_{e}^{*}$ and $\mathbf{q}_{\mathbf{c}}^{*}$ in the last two variables, the weak convergence results (4.25) and (4.31) and the strong convergence result (4.33) and (4.34) it is not difficult to deduce the validity of (3.10)-(3.11).

Finally, we show the attainment of $e_{0}$ and the validity of the entropy inequality (3.8). To do so, we set $u=\chi_{(0, t)} v$ in $(3.8)_{n}$ with arbitrary nonnegative $v \in W^{1 \infty}(\Omega)$ and after integration over $(0, T)$ we get

$$
\begin{aligned}
& \int_{\Omega}\left(s^{n}(t)-s_{0}^{n}\right) v d x-\int_{0}^{t}\left(s^{n} \boldsymbol{v}^{n}+\frac{\mathbf{q}_{e}^{n}}{\theta^{n}}-\mathbf{q}_{\mathfrak{c}}^{n} \zeta^{n}, \nabla v\right) d \tau \\
& \quad \geq \int_{0}^{t}\left(\frac{\mathbf{S}^{n} \cdot \nabla \boldsymbol{v}^{n}}{\theta^{n}}, v\right)+\left(\mathbf{q}_{e}^{n} \cdot \nabla \frac{1}{\theta^{n}}-\mathbf{q}_{\mathfrak{c}}^{n} \cdot \nabla \zeta^{n}, v\right)-\left(\mathfrak{r}^{n} \cdot \zeta^{n}, v\right) d \tau \geq 0 .
\end{aligned}
$$

Next, combining (4.30), (4.31), (4.33) and the continuity of $\zeta^{*}$ we see that

$$
\mathbf{q}_{\mathfrak{c}}^{n} \zeta^{n} \rightarrow \mathbf{q}_{\mathfrak{c}} \zeta \text { weakly in } L^{1}\left(0, T ; L^{1}\left(\Omega ; \mathbb{R}^{d}\right)\right) .
$$

Consequently, using also (4.36), (4.37), (4.32) we can easily let $n \rightarrow \infty$ in the second term on the left hand side of (4.47). Moreover, using (4.6), the strong convergence results (4.33), (4.34), the structural assumption on the entropy $s^{*}(2.38)$, the fact that $s_{\mathfrak{c}}^{*}$ is bounded and the strong convergence of the initial data (3.17), we can use the Fatou lemma to pass to the limit also in the first term on the left hand side of (4.47) with the inequality sign. In addition, having all convergence results above, we can use the Fatou lemma and also the weak lower semicontinuity of norms to pass to the limit also in terms on the right hand side with inequality sign and consequently to obtain (3.8). Moreover, neglecting the nonnegative terms on the right hand side we also have

$$
\int_{\Omega}\left(s^{*}(e(t), \mathfrak{c}(t))-s^{*}\left(e_{0}, \mathfrak{c}_{0}\right)\right) v d x-\int_{0}^{t}\left(s \boldsymbol{v}+\frac{\mathbf{q}_{e}}{\theta}-\mathbf{q}_{\mathbf{c}} \zeta, \nabla v\right) d \tau \geq 0 .
$$

In addition, using the fact that $\mathfrak{c}_{0}$ is already attained, and the assumption on $s_{c}^{*}$ the relation (4.48) implies that

$$
\liminf _{t \rightarrow 0_{+}} \int_{\Omega}\left(s_{e}^{*}(e(t))-s_{e}^{*}\left(e_{0}\right)\right) v d x \geq 0
$$

for all nonnegative $v \in W^{1, \infty}(\Omega)$ and consequently also for all nonnegative $v \in \mathcal{C}(\bar{\Omega})$. Similarly, having (4.46), we can first deduce that

$$
e \in \mathcal{C}\left([0, T] ;\left(W^{2, d}(\Omega)^{*}\right)\right)
$$

and using also (4.45), we can let $n \rightarrow \infty$ in the weak formulation $(3.7)_{n}$ to get

$$
\int_{\Omega}\left(e(t)-e_{0}\right) v d x=\int_{0}^{t}\left(e \boldsymbol{v}+\mathbf{q}_{e}, \nabla v\right)+(\mathbf{S}, \mathbf{D}(\boldsymbol{v}) v) d \tau .
$$

Consequently, we see that

$$
\lim _{t \rightarrow 0_{+}} \int_{\Omega}\left(e(t)-e_{0}\right) v d x=0
$$

for all $v \in \mathcal{C}(\bar{\Omega})$. But since $e_{0} \in L^{1}(\Omega)$ and $e$ is nonnegative, we conclude that

$$
e(t) \rightarrow e_{0} \quad \text { weakly in } L^{1}(\Omega)
$$

as $t \rightarrow 0_{+}$. Indeed, from (4.50) it follows that

$$
e(t) \rightarrow^{*} e_{0} \quad \text { weakly* in } \mathcal{M}(\Omega) .
$$


Moreover, it follows from (4.38) and (4.52) that by using the Biting lemma, we can extract a subsequence (which we do not relabel) and we can also find a nondecreasing sequence $\Omega_{k} \subset$ $\Omega_{k+1} \subset \Omega$ such that

$$
e(t) \rightarrow e_{0} \quad \text { weakly in } L^{1}\left(\Omega_{k}\right)
$$

and for any $\varepsilon>0$ there exists $k$ such that $\left|\Omega \backslash \Omega_{k}\right| \leq \varepsilon$. Thus, for any $u \in L^{\infty}(\Omega)$ we have

$$
\begin{aligned}
\lim _{t \rightarrow 0_{+}}\left(e(t)-e_{0}, u\right) & =\lim _{t \rightarrow 0_{+}} \int_{\Omega \backslash \Omega_{k}} e(t) u d x+\lim _{t \rightarrow 0_{+}} \int_{\Omega_{k}}\left(e(t)-e_{0}\right) u d x-\int_{\Omega \backslash \Omega_{k}} e_{0} u d x \\
& =\lim _{t \rightarrow 0_{+}} \int_{\Omega \backslash \Omega_{k}} e(t) u d x-\int_{\Omega \backslash \Omega_{k}} e_{0} u d x .
\end{aligned}
$$

Finally, using the nonnegativity of $e$ and the fact that $e_{0} \in L^{1}(\Omega)$ we see that

$$
\left|\lim _{t \rightarrow 0_{+}}\left(e(t)-e_{0}, u\right)\right| \leq C(u)\left|\lim _{t \rightarrow 0_{+}} \int_{\Omega \backslash \Omega_{k}} e(t) d x\right|+\delta(k), \quad \delta(k) \stackrel{k \rightarrow \infty}{\rightarrow} 0 .
$$

Thus, using (4.50) with $v \equiv 1$, and the weak convergence of $e(t)$ on $\Omega_{k}$, we see that

$$
\begin{aligned}
\lim _{t \rightarrow 0_{+}} \int_{\Omega \backslash \Omega_{k}} e(t) d x & =\lim _{t \rightarrow 0_{+}} \int_{\Omega} e(t) d x-\lim _{t \rightarrow 0_{+}} \int_{\Omega_{k}} e(t) d x=\int_{\Omega} e_{0} d x-\int_{\Omega_{k}} e_{0} d x \\
& =\int_{\Omega \backslash \Omega_{k}} e_{0} d x \leq \delta(k) .
\end{aligned}
$$

Thus, (4.51) holds at least for a subsequence. But since the whole sequence already converges due to $(4.52)$ we see that $(4.51)$ necessarily holds.

To get also the strong convergence result we use (4.49). Since $s_{e}^{*}$ is strictly concave, the above weak convergence and the inequality (4.49) implies that necessarily

$$
e(t) \rightarrow e_{0} \quad \text { a.e. in } \Omega \text {. }
$$

Consequently, we get that

$$
\lim _{t \rightarrow 0_{+}}\left\|e(t)-e_{0}\right\|_{1}=0,
$$

which finishes the proof of Theorem 3.1.

\section{Proof of Theorem 3.2}

This section is devoted to the proof of the main theorem of this paper. In the previous subsection, we have already observed, that having a priori estimates, it is not difficult to deduce weak sequential compactness. However, for having a priori estimates we need to chose a very special test function, which makes the proof of the existence indeed very complicated. Thus, inspired by [3], we introduce a cascade of Galerkin approximative problems and a cascade of the truncation functions that enable us to get the desired a priori estimate.

First, we introduce the approximative entropies that enable us to get the a priori estimates for the approximative problem with no difficulties. Thus, for arbitrary $\varepsilon, \delta \in(0,1)$ we introduce the following approximations. First, in order to guarantee nonnegativity of each $c_{i}$, we introduce the following $\varepsilon$-approximation of the entropy $s_{\mathfrak{c}}$ as

$$
s_{\mathfrak{c}}^{*, \varepsilon}(\mathfrak{c}):=s_{\mathfrak{c}}^{*}(\mathfrak{c})+\varepsilon \sum_{i=1}^{L} \ln c_{i} .
$$

Note here, that if we control $\left|s^{*, \varepsilon}\right|$ then it directly follows that $c_{i}>0$ almost everywhere in $Q$. Since such approximation in fact makes the problem even more difficult, we mollify $s^{*, \varepsilon}$ and define $s_{\mathfrak{c}}^{\varepsilon, \delta} \in \mathcal{C}^{2}\left(\mathbb{R}^{L} ; \mathbb{R}\right)$ as

$$
s_{\mathfrak{c}}^{*, \varepsilon, \delta}(\mathfrak{c}):= \begin{cases}s_{\mathfrak{c}}^{*, \varepsilon}(\mathfrak{c}) & \text { if for all } i=1, \ldots, L \text { there holds } \delta \leq c_{i} \leq \frac{2}{\delta} \\ \text { concave } & \text { otherwise }\end{cases}
$$


such that for all $\mathfrak{c} \in \mathbb{R}^{L}$

$$
\begin{aligned}
-C(\delta)\left(|\mathfrak{c}|^{2}+1\right) \leq s_{\mathfrak{c}}^{*, \varepsilon, \delta}(\mathfrak{c}) & \leq-C^{-1}(\delta)|\mathfrak{c}|^{2}+C(\delta), \\
\left|\partial_{c_{i} c_{j}}^{2} s_{\mathfrak{c}}^{*, \varepsilon, \delta}(\mathfrak{c})\right|(1+|\mathfrak{c}|)+\left|\partial_{\mathfrak{c}} s_{\mathfrak{c}}^{\varepsilon, \delta}(\mathfrak{c})\right| & \leq C(\delta)(1+|\mathfrak{c}|), \\
\partial_{c_{i} c_{j}}^{2} s_{\mathfrak{c}}^{*, \varepsilon, \delta}(\mathfrak{c}) x_{i} x_{j} & \leq-C^{-1}(\delta)|x|^{2} .
\end{aligned}
$$

Consequently, we introduce the notation for the vectors of chemical potential as

$$
\zeta^{*, \varepsilon}(\mathfrak{c}):=-\partial_{\mathfrak{c}} s^{*, \varepsilon}(\mathfrak{c}), \quad \zeta^{*, \varepsilon, \delta}(\mathfrak{c}):=-\partial_{\mathfrak{c}} s_{\mathfrak{c}}^{*, \varepsilon, \delta}(\mathfrak{c}) .
$$

Moreover, similarly as in Lemma 3.5 we can show that there exists a constant $C>0$ independent of $\varepsilon \in(0,1)$ such that for all $\mathfrak{c} \in G$ we have

$$
\left|\zeta^{*, \varepsilon}(\mathfrak{c})\right|^{2} \leq C\left(1+\left|\mathcal{P}_{\ell} \zeta^{*, \varepsilon}(\mathfrak{c})\right|^{2}\right) .
$$

Indeed, assume that $\mathfrak{c} \in G$. Then there must be at least one component that is large or equal to $1 / L$. Thus, for simplicity assume that $c_{L} \geq 1 / L$. Then it follows from Lemma 3.5 that $\left(\zeta^{*}(\mathfrak{c})\right)^{L} \leq C$. However, using the definition of $\zeta^{*, \varepsilon}$ we also get

$$
\left|\left(\zeta^{*, \varepsilon}(\mathfrak{c})\right)^{L}\right|=\left|\left(\zeta^{*}(\mathfrak{c})\right)^{L}-\frac{\varepsilon}{c_{L}}\right| \leq C(L) .
$$

Consequently, repeating the proof of Lemma 3.5 we deduce that

$$
\begin{aligned}
\left|\zeta^{*, \varepsilon}(\mathfrak{c})\right|^{2} & =\left|P_{\ell} \zeta^{*, \varepsilon}(\mathfrak{c})\right|^{2}+\left|\zeta^{*, \varepsilon}(\mathfrak{c})-P_{\ell} \zeta^{*, \varepsilon}(\mathfrak{c})\right|^{2}=\left|P_{\ell} \zeta^{*, \varepsilon}(\mathfrak{c})\right|^{2}+\frac{1}{L}\left(\sum_{i=1}^{L}\left(\zeta^{*, \varepsilon}(\mathfrak{c})\right)^{i}\right)^{2} \\
& \leq\left|P_{\ell} \zeta^{*, \varepsilon}(\mathfrak{c})\right|^{2}+\frac{L-1}{L}\left|\zeta^{*, \varepsilon}(\mathfrak{c})\right|^{2}+C\left|\left(\zeta^{*, \varepsilon}(\mathfrak{c})\right)^{L}\right|\left|\zeta^{*, \varepsilon}(\mathfrak{c})\right|
\end{aligned}
$$

and (5.4) follows from the Young inequality. Moreover, using the definition of the approximation and also using Lemma 3.5 once again, we see that for all $i=1, \ldots, L$ and all $\mathfrak{c} \in G$

$$
\left(\zeta^{*, \varepsilon}(\mathfrak{c})\right)^{i}=\left(\zeta^{*}(\mathfrak{c})\right)^{i}-\frac{\varepsilon}{c_{i}} \leq\left(\zeta^{*}(\mathfrak{c})\right)^{i} \leq C,
$$

which directly implies that

$$
\left|\zeta^{*}(\mathfrak{c})\right| \leq C\left(1+\left|\zeta^{*, \varepsilon}(\mathfrak{c})\right|\right) \quad \text { for all } \mathfrak{c} \in G .
$$

Next, in order to simplify the analysis concerning the temperature $\theta$, we redefine $s_{e}^{*}$ as follows

$$
s_{e}^{*, \delta}(e):= \begin{cases}s_{e}^{*}(e) & \text { if } \delta \leq e \leq \frac{2}{\delta} \\ \text { concave } & \text { otherwise }\end{cases}
$$

such that $s_{e}^{*, \delta} \in \mathcal{C}^{2}(\mathbb{R}$ and

$$
\left|\partial_{e} s_{e}^{*, \delta}\right|+\left|\partial_{e^{2}}^{2} s_{e}^{*, \delta}(e)\right| \leq C(\delta) \quad \text { for all } e \in \mathbb{R} .
$$

Then we naturally set $\theta^{*, \delta}$ as

$$
\theta^{*, \delta}(e):=\frac{1}{\partial_{e} s^{*, \delta}(e)} .
$$

Finally, due to such approximations we need to correct the corresponding terms in the equations and therefore we define

$$
T_{\delta}(s):= \begin{cases}0 & 0 \leq s \leq \delta \\ 1 & 2 \delta \leq s \leq \frac{1}{\delta} \\ 0 & \frac{2}{\delta} \leq s \\ \text { linear } & \text { otherwise }\end{cases}
$$


and we also define the vector-valued analogue of this truncation $\mathcal{T}_{\delta}: \mathbb{R}^{L} \rightarrow \mathbb{R}$ as

$$
\mathcal{T}_{\delta}(\mathfrak{c}):=\prod_{i=1}^{L} T_{\delta}\left(c_{i}\right) .
$$

Then, we are directly led to introduce an approximation to (3.5)-(3.7), where we just replace all fluxes and the source terms by the following

$$
\begin{aligned}
\mathbf{q}_{\mathfrak{c}}^{*, \delta}(e, \theta, \mathfrak{c}, \nabla \zeta, \nabla \theta) & :=\mathcal{T}_{\delta}(\mathfrak{c}) T_{\delta}(e) \mathbf{q}_{\mathfrak{c}}^{*}(\theta, \mathfrak{c}, \nabla \zeta, \nabla \theta), \\
\mathbf{q}_{e}^{*, \delta}(e, \theta, \mathfrak{c}, \nabla \zeta, \nabla \theta) & :=\mathcal{T}_{\delta}(\mathfrak{c}) T_{\delta}(e) \mathbf{q}_{e}^{*}(\theta, \mathfrak{c}, \nabla \zeta, \nabla \theta), \\
\mathfrak{r}^{*, \delta}(e, \theta, \mathfrak{c}, \zeta) & :=\mathcal{T}_{\delta}(\mathfrak{c}) T_{\delta}(e) \mathfrak{r}^{*}(\theta, \mathfrak{c}, \zeta) .
\end{aligned}
$$

Note here, that this is just an idea how to prove the existence and in fact one need to add to the equations some terms that ensure the compactness of the desired quantities. But having (5.8) in mind, we see that we do not deal with troubles in the constitutive equations as $e$ or $c_{i}$ tends to zero or infinity.

5.1. $(m, n, l)$-Galerkin $(\varepsilon, \delta)$-approximation. First, we find a basis of $W^{d+1,2}\left(\Omega ; \mathbb{R}^{d}\right) \cap W_{0, \operatorname{div}}^{1, r}$ consisting of $\left\{\boldsymbol{w}_{i}\right\}_{i=1}^{\infty}$ that is in addition orthogonal in $L^{2}\left(\Omega ; \mathbb{R}^{d}\right)$. Note that from embedding theorem we directly obtain that $\boldsymbol{w}_{i} \in W_{0}^{1, \infty}\left(\Omega ; \mathbb{R}^{d}\right)$. Moreover, due to the density we have that it is also basis of $W_{0, \text { div }}^{1, r}$. The finite dimensional spaces $V^{n}$ is then defined as the linear span of $\left\{\boldsymbol{w}_{i}\right\}_{i=1}^{n}$. Similarly, let $\left\{u_{i}\right\}_{i=1}^{\infty}$ is a basis of $W^{1,2}(\Omega)$, which is orthogonal in $L^{2}$ and also in $W^{1,2}$ and we denote $W^{m}$ the linear span of $\left\{u_{i}\right\}_{i=1}^{m}$. Finally, we denote $P_{1}^{n}$ and $P_{2}^{m}$, the orthogonal projections as $P_{1}^{n}: W^{d+1,2}\left(\Omega ; \mathbb{R}^{d}\right) \cap W_{0, \text { div }}^{1, r} \rightarrow V_{\text {div }}^{n}$ and $P_{2}^{m}: W^{1,2}(\Omega) \rightarrow W^{m}$, respectively.

Finally, we define $(m, n, l, \varepsilon, \delta)$ approximative problem in the following way: for fixed $\varepsilon, \delta \in(0,1)$ and $m, n, l \in \mathbb{N}$ to find

$$
\begin{aligned}
\boldsymbol{v}^{n, m, l, \varepsilon, \delta}(t, x) & :=\sum_{i=1}^{n} \alpha_{i}^{n, m, l, \varepsilon, \delta}(t) \boldsymbol{w}_{i}(x), \\
\mathfrak{c}^{n, m, l, \varepsilon, \delta}(t, x) & :=\sum_{i=1}^{m} \boldsymbol{\beta}_{i}^{n, m, l, \varepsilon, \delta}(t) u_{i}(x), \\
e^{n, m, l, \varepsilon, \delta}(t, x) & :=\sum_{i=1}^{l} \gamma_{i}^{n, m, l, \varepsilon, \delta}(t) u_{i}(x) .
\end{aligned}
$$

where $\alpha_{i}^{n, m, l, \varepsilon, \delta}:[0, T] \rightarrow \mathbb{R}, \boldsymbol{\beta}_{i}^{n, m, l, \varepsilon, \delta}:[0, T] \rightarrow \mathbb{R}^{L}$ and $\gamma_{i}^{n, m, l, \varepsilon, \delta}:[0, T] \rightarrow \mathbb{R}$ are continuous functions with the initial data given by

$$
\boldsymbol{v}^{n, m, l, \varepsilon, \delta}(0):=P_{1}^{n}\left(\boldsymbol{v}_{0}\right), \quad \mathfrak{c}^{n, m, l, \varepsilon, \delta}(0):=\left(P_{2}^{m}\left(c_{0}^{\delta}\right)_{1}, \ldots, P_{2}^{m}\left(c_{0}^{\delta}\right)_{L}\right), \quad e^{n, m, l, \varepsilon, \delta}(0):=P_{3}^{l}\left(e_{0}^{\varepsilon, \delta}\right),
$$

where $\mathfrak{c}_{0}^{\varepsilon, \delta}$ is defined as

$$
\mathfrak{c}_{0}^{\varepsilon, \delta}:=\frac{\mathfrak{c}_{0}}{1+\max (\varepsilon, \delta) L}+\frac{\max (\varepsilon, \delta) \ell}{1+\max (\varepsilon, \delta) L}
$$

and $e_{0}^{\varepsilon, \delta}$ is given by

$$
e_{0}^{\varepsilon}:=\min \left(\varepsilon^{-1}, e_{0}\right)+\max (\varepsilon, \delta) .
$$

Moreover, we require the validity of the following identities:

$$
\begin{gathered}
\left(\boldsymbol{v}_{, t}^{n, m, l, \varepsilon, \delta}, \boldsymbol{w}\right)-\left(\boldsymbol{v}^{n, m, l, \varepsilon, \delta} \otimes \boldsymbol{v}^{n, m, l, \varepsilon, \delta}, \nabla \boldsymbol{w}\right)+\left(\mathbf{S}^{n, m, l, \varepsilon, \delta}, \nabla \boldsymbol{w}\right)=0 \quad \text { for all } \boldsymbol{w} \in V_{\mathrm{div}}^{n}, \\
\left(\mathfrak{c}_{, t}^{n, m, l, \varepsilon, \delta}, \boldsymbol{u}\right)+\varepsilon\left(\nabla \mathfrak{c}^{n, m, l, \varepsilon, \delta}, \nabla \boldsymbol{u}\right)-\left(\mathfrak{c}^{n, m, l, \varepsilon, \delta} \otimes \boldsymbol{v}^{n, m, l, \varepsilon, \delta}, \nabla \boldsymbol{u}\right)-\left(\mathbf{q}_{\mathfrak{c}}^{n, m, l, \varepsilon, \delta}, \nabla \boldsymbol{u}\right) \\
=\left(\mathfrak{r}^{n, m, l, \varepsilon, \delta}, \boldsymbol{u}\right) \quad \text { for all } \boldsymbol{u} \in\left(W^{m}\right)^{L},
\end{gathered}
$$




$$
\begin{array}{rr}
\left(e_{, t}^{n, m, l, \varepsilon, \delta}, u\right)+\varepsilon\left(\nabla e^{n, m, l, \varepsilon, \delta}, \nabla u\right)-\left(e^{n, m, l, \varepsilon, \delta} \boldsymbol{v}^{n, m, l, \varepsilon, \delta}, \nabla u\right)-\left(\mathbf{q}_{e}^{n, m, l, \varepsilon, \delta}, \nabla u\right) \\
=\left(\mathbf{S}^{n, m, l, \varepsilon, \delta} \cdot \nabla \boldsymbol{v}^{n, m, l, \varepsilon, \delta}, u\right) & \text { for all } u \in W^{l},
\end{array}
$$

where (we recall the definition (5.8))

$$
\begin{aligned}
& \mathbf{q}_{\mathfrak{c}}^{n, m, l, \varepsilon, \delta}:=\mathbf{q}_{\mathfrak{c}}^{*, \delta}\left(e^{n, m, l, \varepsilon, \delta}, \theta^{n, m, l, \varepsilon, \delta}, \mathfrak{c}^{n, m, l, \varepsilon, \delta}, \nabla \zeta^{n, m, l, \varepsilon, \delta}, \nabla \theta^{n, m, l, \varepsilon, \delta}\right), \\
& \mathbf{q}_{e}^{n, m, l, \varepsilon, \delta}:=\mathbf{q}_{e}^{*, \delta}\left(e^{n, m, l, \varepsilon, \delta}, \theta^{n, m, l, \varepsilon, \delta}, \mathfrak{c}^{n, m, l, \varepsilon, \delta}, \nabla \zeta^{n, m, l, \varepsilon, \delta}, \nabla \theta^{n, m, l, \varepsilon, \delta}\right), \\
& \mathbf{S}^{n, m, l, \varepsilon, \delta}:=\mathbf{S}^{*}\left(\theta^{n, m, l, \varepsilon, \delta}, \mathfrak{c}^{n, m, l, \varepsilon, \delta}, \mathbf{D}\left(\boldsymbol{v}^{n, m, l, \varepsilon, \delta}\right)\right), \\
& \mathfrak{r}^{n, m, l, \varepsilon, \delta}:=\mathfrak{r}^{*, \delta}\left(e^{n, m, l, \varepsilon, \delta}, \theta^{n, m, l, \varepsilon, \delta}, \mathfrak{c}^{n, m, l, \varepsilon, \delta}, \zeta^{n, m, l, \varepsilon, \delta}\right),
\end{aligned}
$$

and in addition

$$
\begin{aligned}
& \theta^{n, m, l, \varepsilon, \delta}:=\max \left(0, \theta^{*, \delta}\left(e^{n, m, l, \varepsilon, \delta}\right)\right), \\
& \zeta^{n, m, l, \varepsilon, \delta}:=P_{2}^{m}\left(\zeta^{*, \varepsilon, \delta}\left(\mathfrak{c}^{n, m, l, \varepsilon, \delta}\right)\right) .
\end{aligned}
$$

Due to the continuity of all approximative constitutive relations (5.15)-(5.20), the short time existence directly follows from the Carathéodory theory. Moreover, using a priori estimates established below, we can extend the solution onto the whole time interval $(0, T)$. Thus, we continue with the estimates independent of $l$ and with limit $l \rightarrow \infty$. In what follows, we denote by $C$ a constant that is uniform with respect all approximative parameters. Moreover, we will clearly denote any dependence on the approximative parameters in the rest of the proof.

5.2. Limit $l \rightarrow \infty$. This subsection is devoted to the limit passage $l \rightarrow \infty$. For simplicity we avoid writing indexes $m, n, \varepsilon, \delta$ and we keep only the index $l$. However, if some estimate will depend on these parameters we clearly denote it. First, setting $\boldsymbol{w}:=\boldsymbol{v}^{l}$ in (5.12) we deduce the following energy identity

$$
\frac{1}{2} \frac{d}{d t}\left\|\boldsymbol{v}^{l}(t)\right\|_{2}^{2}+\left(\mathbf{S}^{l}, \nabla \boldsymbol{v}^{l}\right)=0 .
$$

Hence, using (2.36) and the Korn inequality we find that

$$
\sup _{t \in(0, T)}\left\|\boldsymbol{v}^{l}(t)\right\|_{2}^{2}+\int_{0}^{T}\left\|\boldsymbol{v}^{l}\right\|_{1, r}^{r}+\left\|\mathbf{S}^{l}\right\|_{r^{\prime}}^{r^{\prime}} d t \leq C .
$$

Note that due to our choice of the basis we can also deduce from (5.22) that

$$
\int_{0}^{T}\left\|\boldsymbol{v}^{l}\right\|_{1,2 r}^{2 r}+\left\|\mathbf{S}^{l}\right\|_{2 r^{\prime}}^{2 r^{\prime}} d t \leq C(n) .
$$

Next, setting $u:=e^{l}$ in (5.14) and using (2.36), we get

$$
\frac{1}{2} \frac{d}{d t}\left\|e^{l}(t)\right\|_{2}^{2}+\varepsilon\left\|\nabla e^{l}\right\|_{2}^{2}-\left(\mathbf{q}_{e}^{l}, \nabla e^{l}\right) \leq C\left\|e^{l}\right\|_{2}\left(1+\left\|\boldsymbol{v}^{l}\right\|_{2 r}^{r}\right) .
$$

Moreover, using (A3), the fact that $\theta^{*, \delta}$ is a nondecreasing function of $e$ and the presence of cut-off functions $T_{\delta}$ and $\mathcal{T}_{\delta}$ in definition of $\mathbf{q}_{e}^{*, \delta}$ (see (5.8)), we can estimate the third term on the left hand side with the help of the Young inequality as

$$
-\left(\mathbf{q}_{e}^{l}, \nabla e^{l}\right) \geq-\frac{\varepsilon}{2}\left\|\nabla e^{l}\right\|_{2}^{2}-C(\varepsilon, \delta)\left\|\nabla \mathfrak{c}^{l}\right\|_{2}^{2} .
$$

Thus, inserting (5.25) into (5.24) we get

$$
\frac{d}{d t}\left\|e^{l}(t)\right\|_{2}^{2}+\varepsilon\left\|\nabla e^{l}\right\|_{2}^{2} \leq C\left\|e^{l}\right\|_{2}\left(1+\left\|\boldsymbol{v}^{l}\right\|_{2 r}^{r}\right)+C(\varepsilon, \delta)\left\|\nabla \mathfrak{c}^{l}\right\|_{2}^{2} .
$$

Finally, setting $\boldsymbol{u}:=\mathfrak{c}^{l}$ in (5.13), using (5.16), the assumption (A2), the presence of cut-off functions in the definition of $\mathbf{q}_{\mathbf{c}}^{*, \delta}$ (see the definition (5.8)) and the continuity of the projection $P_{2}^{m}$, we immediately deduce with the help of the Young inequality that

$$
\frac{d}{d t}\left\|\mathfrak{c}^{l}(t)\right\|_{2}^{2} \leq \frac{\varepsilon}{2}\left\|\nabla e^{l}\right\|_{2}^{2}+C(\varepsilon, \delta)\left\|\nabla \mathfrak{c}^{l}\right\|_{2}^{2} .
$$


Next, using the fact that all norms on finite dimensional spaces are equivalent, in particular the following inequality

$$
\left\|\nabla \mathfrak{c}^{l}\right\|_{2}^{2} \leq C(m)\left\|\mathfrak{c}^{l}\right\|_{2}^{2}
$$

and summing (5.26) and (5.27), we obtain

$$
\begin{aligned}
& \frac{d}{d t}\left(\left\|e^{l}(t)\right\|_{2}^{2}+\left\|\mathfrak{c}^{l}(t)\right\|_{2}^{2}\right)+\varepsilon\left\|\nabla e^{l}\right\|_{2}^{2} \\
& \quad \leq C\left\|\nabla \boldsymbol{v}^{l}\right\|_{2 r}^{2 r}+C(\varepsilon, \delta, n, m)\left(\left\|e^{l}\right\|_{2}^{2}+\left\|\mathfrak{c}^{l}(t)\right\|_{2}^{2}\right) .
\end{aligned}
$$

Consequently, the Gronwall inequality implies that

$$
\sup _{t \in(0, T)}\left(\left\|\mathfrak{c}^{l}(t)\right\|_{2}^{2}+\left\|e^{l}(t)\right\|_{2}^{2}\right)+\int_{0}^{T}\left\|e^{l}\right\|_{1,2}^{2} d t \leq C(\varepsilon, \delta, n, m) .
$$

In addition, using (5.15), (5.16) and keeping in mind the presence of cut-off functions, we can deduce that

$$
\int_{0}^{T}\left\|\mathbf{q}_{e}^{l}\right\|_{2}^{2}+\left\|\mathbf{q}_{\mathfrak{c}}^{l}\right\|_{2}^{2} d t \leq C(n, m, \varepsilon, \delta)
$$

Finally, using (5.22), (5.30) and (5.28), we can observe from (5.12)-(5.14) that

$$
\int_{0}^{T}\left\|\mathfrak{c}_{, t}^{l}\right\|_{2}^{2}+\left\|\boldsymbol{v}_{, t}^{l}\right\|_{2}^{2}+\left\|e_{, t}^{l}\right\|_{-1,2}^{2} d t \leq C(\varepsilon, \delta, n, m) .
$$

Thus, it follows from $(5.22),(5.30),(5.28),(5.31),(5.32)$ and the Aubin-Lions lemma that we can extract a subsequence that we do not relabel such that

$$
\begin{gathered}
\boldsymbol{v}^{l} \rightarrow \boldsymbol{v} \\
\boldsymbol{v}^{l} \rightarrow \boldsymbol{v} \\
\mathfrak{c}^{l} \rightarrow \mathfrak{c} \\
\mathfrak{c}^{l} \rightarrow \mathfrak{c} \\
e^{l} \rightarrow e \\
e^{l} \rightarrow e \\
\mathbf{q}_{e}^{l} \rightarrow \mathbf{q}_{e} \\
\mathbf{q}_{\mathfrak{c}}^{l} \rightarrow \mathbf{q}_{\mathfrak{c}} \\
\mathbf{S}^{l} \rightarrow \mathbf{S} \\
\mathfrak{r}^{l} \rightarrow \mathfrak{r}
\end{gathered}
$$

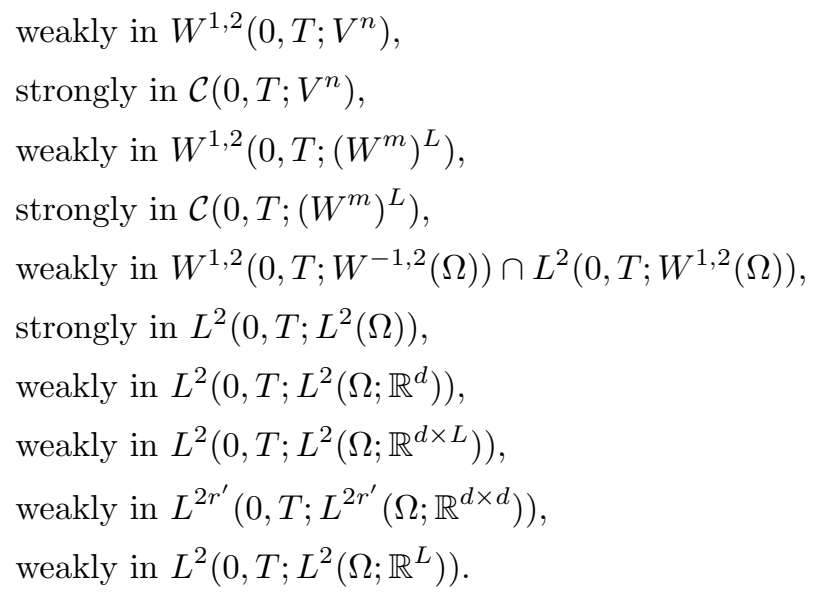

Thus, using (5.2), (5.3), (5.6), (5.7), (5.36), (5.38) and the continuity of the projection $P_{2}^{m}$ we see that

$$
\begin{aligned}
& \theta^{l} \rightarrow \theta \\
& \theta^{l} \rightarrow \theta \\
& \zeta^{l} \rightarrow \zeta
\end{aligned}
$$

weakly in $L^{2}\left(0, T ; W^{1,2}(\Omega)\right)$, strongly in $L^{2}\left(0, T ; L^{2}(\Omega)\right)$, strongly in $\mathcal{C}\left(0, T ; W^{1,2}\left(\Omega ; \mathbb{R}^{L}\right)\right)$,

where

$$
\begin{aligned}
\theta & :=\max \left(0, \theta^{*, \delta}(e)\right), \\
\zeta & :=P_{2}^{m}\left(\zeta^{*, \varepsilon, \delta}(\mathfrak{c})\right)
\end{aligned}
$$


Having all above convergence results and using the linear dependence of $\mathbf{q}_{e}^{l}$ and $\mathbf{q}_{e}^{l}$ on $\nabla \theta^{l}$, we can easily identify the limit in all fluxes to deduce that

$$
\begin{aligned}
\mathbf{q}_{\mathfrak{c}} & =\mathbf{q}_{\mathfrak{c}}^{*, \delta}(\theta, \mathfrak{c}, \nabla \zeta, \nabla \theta), \\
\mathbf{q}_{e} & =\mathbf{q}_{e}^{*, \delta}(\theta, \mathfrak{c}, \nabla \zeta, \nabla \theta), \\
\mathbf{S} & =\mathbf{S}^{*}(\theta, \mathfrak{c}, \mathbf{D}(\boldsymbol{v})), \\
\mathfrak{r} & =\mathfrak{r}^{*, \delta}(\theta, \mathfrak{c}, \zeta) .
\end{aligned}
$$

In addition, it is the also easy to let $l \rightarrow \infty$ in (5.12)-(5.14) to conclude

$$
\begin{gathered}
\left(\boldsymbol{v}_{, t}, \boldsymbol{w}\right)-(\boldsymbol{v} \otimes \boldsymbol{v}, \nabla \boldsymbol{w})+\left(\mathbf{S}^{\prime} \nabla \boldsymbol{w}\right)=0 \quad \text { for all } \boldsymbol{w} \in V_{\mathrm{div}}^{n} . \\
\left(\mathfrak{c}_{, t}, \boldsymbol{u}\right)+\varepsilon(\nabla \mathfrak{c}, \nabla \boldsymbol{u})-(\mathfrak{c} \otimes \boldsymbol{v}, \nabla \boldsymbol{u})-\left(\mathbf{q}_{\mathfrak{c}}, \nabla \boldsymbol{u}\right)=(\mathfrak{r}, \boldsymbol{u}) \quad \text { for all } \boldsymbol{u} \in\left(W^{m}\right)^{L} \\
\left(e_{, t}, u\right)+\varepsilon(\nabla e, \nabla u)-(e \boldsymbol{v}, \nabla u)-\left(\mathbf{q}_{e}, \nabla u\right)=(\mathbf{S} \cdot \nabla \boldsymbol{v}, u) \\
\quad \text { for all } u \in W^{1,2}(\Omega) \text { and a.a. } t \in(0, T) .
\end{gathered}
$$

Moreover, it is standard to prove at this level the attainment of initial conditions and we have

$$
\boldsymbol{v}(0)=P_{1}^{n}\left(\boldsymbol{v}_{0}\right), \quad \mathfrak{c}(0)=P_{2}^{m}\left(\mathfrak{c}_{0}^{\varepsilon, \delta}\right), \quad e(0)=e_{0}^{\varepsilon, \delta} .
$$

5.3. Limit $m \rightarrow \infty$. This section is devoted to the limit passage $m \rightarrow \infty$. Thus, we denote the solution constructed in the previous subsection by superscript $m$ and omit writing all other superscripts for simplicity. Before we let $m \rightarrow \infty$, we recover the minimum principle for the internal energy $e^{m}$. Hence, setting $u:=\min \left(0, e^{m}-\delta\right)$ in (5.54) we see due to the presence of $T_{\delta}\left(e^{m}\right)$ in $\mathbf{q}_{e}^{m}$ and due to the nonpositivity of the right hand side gurantueed by (2.36) that

$$
\frac{d}{d t}\left\|\min \left(0, e^{m}-\delta\right)\right\|_{2}^{2} \leq 0 .
$$

Thus, using (5.55), we directly obtain that

$$
e^{n, m} \geq \delta \quad \text { almost everywhere in } Q .
$$

Consequently, it follows from the definition of $s_{e}^{*, \delta}$ and (5.7) that we can replace (5.46) by

$$
\theta^{m}:=\theta^{*}\left(e^{m}\right)
$$

without any change in (5.52)-(5.54).

Next, we derive $m$-independent estimate that will help us to let $m \rightarrow \infty$. First, we can follow the procedure from the previous subsection to easily derive the following estimates

$$
\begin{aligned}
\sup _{t \in(0, T)}\left\|\boldsymbol{v}^{m}(t)\right\|_{2}^{2}+\int_{0}^{T}\left\|\boldsymbol{v}^{m}\right\|_{1, r}^{r}+\left\|\mathbf{S}^{m}\right\|_{r^{\prime}}^{r^{\prime}} d t \leq C . \\
\int_{0}^{T}\left\|\boldsymbol{v}_{, t}^{m}\right\|_{2}^{2}+\left\|\boldsymbol{v}^{m}\right\|_{1,2 r}^{2 r}+\left\|\mathbf{S}^{m}\right\|_{2 r^{\prime}}^{2 r^{\prime}} d t \leq C(n) .
\end{aligned}
$$

Next, we set $u:=1$ in (5.54) and with the help of (5.58) we see that

$$
\sup _{t \in(0, T)}\left\|e^{m}(t)\right\|_{1} \leq C+\left\|e^{m}(0)\right\|_{1} \leq C,
$$

where the last inequality follows from (5.55) provided that $\varepsilon, \delta \leq 1$. Next, since $e^{m} \geq \delta$ we have due to (2.39) that also $\theta^{m} \geq C_{2}^{-1} \delta$ and consequently we can set

$$
u:=\frac{1}{\theta^{m}}
$$


in (5.54) and after using (5.49) and (2.33) we observe (note that we use here that $\operatorname{div} \boldsymbol{v}^{m}=0$ which leads to the fact that the convective term vanishes)

$$
\begin{array}{r}
\frac{d}{d t} \int_{\Omega} s_{e}^{*}\left(e^{m}\right) d x=-\varepsilon \int_{\Omega} \partial_{e^{2}}^{2} s_{e}^{*}\left(e^{m}\right)\left|\nabla e^{m}\right|^{2} d x+\int_{\Omega} \frac{\mathbf{S}^{m} \cdot \mathbf{D}\left(\boldsymbol{v}^{m}\right)}{\theta^{m}} d x \\
\quad+\int_{\Omega} \mathcal{T}_{\delta}\left(\mathfrak{c}^{m}\right) T_{\delta}\left(e^{m}\right) \frac{\kappa\left(\mathfrak{c}^{m}, \theta^{m}\right)}{\left(\theta^{m}\right)^{2}}\left|\nabla \theta^{m}\right|^{2} d x \\
-\left(\mathcal{T}_{\delta}\left(\mathfrak{c}^{m}\right) T_{\delta}\left(e^{m}\right) \nabla \zeta^{m}, \nabla \frac{1}{\theta^{m}} \otimes \mathfrak{m}\left(\mathfrak{c}^{m}, \theta^{m}\right)\right) .
\end{array}
$$

Similarly, setting $\boldsymbol{u}:=-\zeta^{m}$ in (5.53) (note that it is a possible setting due to the defintion of $\zeta^{m}$, where the projection $P_{2}^{m}$ palys the crucial role) we get

$$
\begin{aligned}
-\left(\mathfrak{c}_{, t}^{m}, P_{2}^{m}\left(\zeta^{*, \varepsilon, \delta}\left(\mathfrak{c}^{m}\right)\right)\right)-\varepsilon\left(\nabla \mathfrak{c}^{m}, \nabla P_{2}^{m}\left(\zeta^{*, \varepsilon, \delta}\left(\mathfrak{c}^{m}\right)\right)+\left(\mathfrak{c}^{m} \otimes \boldsymbol{v}^{m}, \zeta^{m}\right)\right. \\
+\left(\mathbf{q}_{\mathfrak{c}}^{m}, \nabla P_{2}^{m} \zeta^{*, \varepsilon, \delta}\left(\mathfrak{c}^{m}\right)\right)=-\left(\mathfrak{r}^{m}, \zeta^{m}\right) .
\end{aligned}
$$

First, using the orthogonality of the basis and also (5.3) we obtain

$$
\begin{aligned}
& -\left(\mathfrak{c}_{, t}^{m}, P_{2}^{m} \zeta^{*, \varepsilon, \delta}\left(\mathfrak{c}^{m}\right)\right)-\varepsilon\left(\nabla \mathfrak{c}^{m}, \nabla P_{2}^{m} \zeta^{*, \varepsilon, \delta}\left(\mathfrak{c}^{m}\right)\right) \\
& \quad=-\left(\mathfrak{c}_{, t}^{m}, \zeta^{*, \varepsilon, \delta}\left(\mathfrak{c}^{m}\right)\right)-\varepsilon\left(\nabla \mathfrak{c}^{m}, \nabla \zeta^{*, \varepsilon, \delta}\left(\mathfrak{c}^{m}\right)\right) \\
& \quad=\frac{d}{d t} \int_{\Omega} s^{*, \varepsilon, \delta}\left(\mathfrak{c}^{m}\right) d x+\varepsilon\left(\partial_{\mathfrak{c}^{2}}^{2} s_{\mathfrak{c}}^{*, \varepsilon, \delta}\left(\mathfrak{c}^{m}\right) \nabla \mathfrak{c}^{m}, \nabla \mathfrak{c}^{m}\right) .
\end{aligned}
$$

Similarly, using the Hölder inequality, the continuity of the projections $P_{2}^{m}$ and (5.2)-(5.3), we deduce

$$
\begin{aligned}
\left|\left(\mathfrak{c}^{m} \otimes \boldsymbol{v}^{m}, \nabla \zeta^{m}\right)\right| & \leq\left\|\boldsymbol{v}^{m}\right\|_{\infty}\|\| \mathfrak{c}^{m}\left\|_{2}\right\| \nabla \zeta^{m} \|_{2} \\
& \leq C\left\|\boldsymbol{v}^{m}\right\|_{\infty}\left\|\mathfrak{c}^{m}\right\|_{2}\left\|\nabla \zeta^{*, \varepsilon, \delta}\left(\mathfrak{c}^{m}\right)\right\|_{2} \\
& \leq C(\varepsilon, \delta)\left\|\boldsymbol{v}^{m}\right\|_{\infty}\left(1+\left\|s_{\mathfrak{c}}^{*, \varepsilon, \delta}\left(\mathfrak{c}^{m}\right)\right\|_{1}^{\frac{1}{2}}\right)\left(1+\left\|\nabla \mathfrak{c}^{m}\right\|_{2}\right)
\end{aligned}
$$

Finally, for the term with $\mathbf{q}_{\mathfrak{c}}$ we use (5.48), (2.24) and (2.30) which lead to the following inequality

$$
\begin{aligned}
\left(\mathbf{q}_{\mathfrak{c}}^{m}, \nabla \zeta^{m}\right)= & -\left(\mathcal{T}_{\delta}\left(\mathfrak{c}^{m}\right) T_{\delta}\left(e^{m}\right) M\left(\theta^{m}, \mathfrak{c}^{m}\right) \nabla \zeta^{m}, \nabla \zeta^{m}\right) \\
& \left.-\left(\mathcal{T}_{\delta}\left(\mathfrak{c}^{m}\right) T_{\delta}\left(e^{m}\right) \nabla \zeta^{m}\right), \mathfrak{m}\left(\mathfrak{c}^{m}, \theta^{m}\right) \otimes \nabla \frac{1}{\theta^{m}}\right) \\
\leq & -\left(\mathcal{T}_{\delta}\left(\mathfrak{c}^{m}\right) T_{\delta}\left(e^{m}\right) \nabla \zeta^{m}, \mathfrak{m}\left(\mathfrak{c}^{m}, \theta^{m}\right) \otimes \nabla \frac{1}{\theta^{m}}\right) .
\end{aligned}
$$

Thus, substituting (5.63)-(5.65) to (5.62) and for the term on the right hand side using (2.28), we get

$$
\begin{array}{r}
\frac{d}{d t} \int_{\Omega} s_{\mathfrak{c}}^{*, \varepsilon, \delta}\left(\mathfrak{c}^{m}\right) d x+C(\varepsilon, \delta)\left\|\boldsymbol{v}^{m}\right\|_{\infty}\left(1+\left\|s_{\mathfrak{c}}^{*, \varepsilon, \delta}\left(\mathfrak{c}^{m}\right)\right\|_{1}^{\frac{1}{2}}\right)\left(1+\left\|\nabla \mathfrak{c}^{m}\right\|_{2}\right) \\
\geq\left(\mathcal{T}_{\delta}\left(\mathfrak{c}^{m}\right) T_{\delta}\left(e^{m}\right) \nabla \zeta^{m}, \mathfrak{m}\left(\mathfrak{c}^{m}, \theta^{m}\right) \otimes \nabla \frac{1}{\theta^{m}}\right)+C(\delta, \varepsilon)\left\|\nabla \mathfrak{c}^{m}\right\|_{2}^{2}
\end{array}
$$

To finish the estimates based on the entropy balance we sum (5.61) and (5.66), neglect the terms having the correct signs and with the help of the Young inequality applied onto the convective term we observe

$$
\begin{array}{r}
C(\varepsilon, \delta)\left(1+\left\|e^{m}\right\|_{1}+\left\|\boldsymbol{v}^{m}\right\|_{\infty}^{2}\right)\left(C(\varepsilon, \delta)-\int_{\Omega} s_{\mathfrak{c}}^{*, \varepsilon, \delta}\left(\mathfrak{c}^{m}\right)+s_{e}^{*}\left(e^{m}\right) d x\right) \\
\geq-\frac{d}{d t} \int_{\Omega} s_{\mathfrak{c}}^{*, \varepsilon, \delta}\left(\mathfrak{c}^{m}\right)+s_{e}^{*}\left(e^{m}\right) d x+C(\delta, \varepsilon)\left\|\nabla \mathfrak{c}^{m}\right\|_{2}^{2} .
\end{array}
$$

Hence, using (5.2) and a priori estimates (5.58)-(5.60) (and also the embedding $W^{1,2 r} \hookrightarrow L^{\infty}$ ), we deduce that

$$
\sup _{t \in(0, T)}\left\|\mathfrak{c}^{m}\right\|_{2}^{2}+\int_{0}^{T}\left\|\nabla \mathfrak{c}^{m}\right\|_{2}^{2} d t \leq C(\varepsilon, \delta, n) .
$$


Thus, having such an estimate, we can set $u:=e^{m}$ in (5.54) and by a standard manipulation and using the defintion of $\mathbf{q}_{e}^{m}$ and the setimate above, we get

$$
\sup _{t \in(0, T)}\left\|e^{m}\right\|_{2}^{2}+\int_{0}^{T}\left\|\nabla e^{m}\right\|_{2}^{2} d t \leq C(\varepsilon, \delta, n) .
$$

Consequently, it is now standard to deduce that also

$$
\int_{0}^{T}\left\|\mathfrak{c}_{, t}^{m}\right\|_{-1,2}^{2}+\left\|\boldsymbol{v}_{, t}^{m}\right\|_{2}^{2}+\left\|e_{, t}^{m}\right\|_{-1,2}^{2} d t \leq C(\varepsilon, \delta, n) .
$$

Thus, having (5.58)-(5.59) and (5.68)-(5.70), we can extract a non relabeled subsequence and by using the Aubin-Lions lemma we get

$(5.71) \quad \boldsymbol{v}^{m} \rightarrow \boldsymbol{v}$

$(5.72) \quad \boldsymbol{v}^{m} \rightarrow \boldsymbol{v}$

$$
\mathfrak{c}^{m} \rightarrow^{*} \mathfrak{c}
$$$$
\mathfrak{c}^{m} \rightarrow \mathfrak{c}
$$$$
e^{m} \rightarrow e
$$$$
e^{m} \rightarrow e
$$

$$
\mathbf{q}_{e}^{m} \rightarrow \mathbf{q}_{e}
$$$$
\mathbf{q}_{\mathrm{c}}^{m} \rightarrow \mathbf{q}_{\mathrm{c}}
$$

$$
\mathbf{S}^{m} \rightarrow \mathbf{S}
$$$$
\mathfrak{r}^{l} \rightarrow \mathfrak{r}
$$

weakly in $W^{1,2}\left(0, T ; V^{n}\right)$,

strongly in $\mathcal{C}\left(0, T ; V^{n}\right)$,

weakly in $L^{2}\left(0, T ; W^{1,2}\left(\Omega ; \mathbb{R}^{L}\right)\right) \cap W^{1,2}\left(0, T ; W^{-1,2}\left(\Omega ; \mathbb{R}^{L}\right)\right)$,

strongly in $L^{2}\left(0, T ; L^{2}\left(\Omega ; \mathbb{R}^{L}\right)\right)$,

weakly in $W^{1,2}\left(0, T ; W^{-1,2}(\Omega)\right) \cap L^{2}\left(0, T ; W^{1,2}(\Omega)\right)$,

strongly in $L^{2}\left(0, T ; L^{2}(\Omega)\right)$,

weakly in $L^{2}\left(0, T ; L^{2}\left(\Omega ; \mathbb{R}^{d}\right)\right)$,

weakly in $L^{2}\left(0, T ; L^{2}\left(\Omega ; \mathbb{R}^{d \times L}\right)\right)$,

weakly in $L^{q^{\prime}}\left(0, T ; L^{q^{\prime}}\left(\Omega ; \mathbb{R}^{d \times d}\right)\right)$,

weakly in $L^{2}\left(0, T ; L^{2}\left(\Omega ; \mathbb{R}^{L}\right)\right)$.

Thus, using (5.76) and (5.7), (5.3), (5.73)-(5.74) and the continuity of the projections $P_{2}^{m}$ we have

$$
\begin{aligned}
& \theta^{m} \rightarrow \theta \\
& \theta^{m} \rightarrow \theta \\
& \zeta^{m} \rightarrow \zeta
\end{aligned}
$$
weakly in $L^{2}\left(0, T ; W^{1,2}(\Omega)\right)$, strongly in $L^{2}\left(0, T ; L^{2}(\Omega)\right)$,

where

$$
\begin{aligned}
& \theta=\theta^{*}(e), \\
& \zeta=\zeta^{*, \varepsilon, \delta}(\mathfrak{c}) .
\end{aligned}
$$

Having all above convergence results and using the linear dependence of $\mathbf{q}_{e}^{m}$ and $\mathbf{q}_{\mathbf{c}}^{m}$ on $\nabla \theta^{m}$ and $\nabla \zeta^{m}$, using the fact that $V^{n}$ is finite dimensional, we can easily let $m \rightarrow \infty$ in (5.52)-(5.54) to conclude

$$
\begin{array}{r}
\left(\boldsymbol{v}_{, t}, \boldsymbol{w}\right)-(\boldsymbol{v} \otimes \boldsymbol{v}, \nabla \boldsymbol{w})+(\mathbf{S}, \nabla \boldsymbol{w})=0 \quad \text { for all } \boldsymbol{w} \in V_{\mathrm{div}}^{n}, \\
\left\langle\mathfrak{c}_{, t}, \boldsymbol{u}\right\rangle+\varepsilon(\nabla \mathfrak{c}, \nabla \boldsymbol{u})-(\mathfrak{c} \otimes \boldsymbol{v}, \nabla \boldsymbol{u})-\left(\mathbf{q}_{\mathfrak{c}}, \nabla \boldsymbol{u}\right)=(\mathfrak{r}, \boldsymbol{u}) \\
\text { for all } \boldsymbol{u} \in W^{1,2}\left(\Omega ; \mathbb{R}^{L}\right) \text { and a.a. } t \in(0, T), \\
\left\langle e_{, t}, u\right\rangle+\varepsilon(\nabla e, \nabla u)-(e \boldsymbol{v}, \nabla u)-\left(\mathbf{q}_{e}, \nabla u\right)=(\mathbf{S} \cdot \nabla \boldsymbol{v}, u) \\
\text { for all } u \in W^{1,2}(\Omega) \text { and a.a. } t \in(0, T),
\end{array}
$$

where

$$
\begin{aligned}
\mathbf{q}_{\mathfrak{c}} & =\mathbf{q}_{\mathfrak{c}}^{*, \delta}(\theta, \mathfrak{c}, \nabla \zeta, \nabla \theta), \\
\mathbf{q}_{e} & \left.=\mathbf{q}_{e}^{*, \delta}(\theta, \mathfrak{c}, \nabla \zeta(\mathfrak{c})), \nabla \theta\right), \\
\mathbf{S} & =\mathbf{S}^{*}(\theta, \mathfrak{c}, \mathbf{D}(\boldsymbol{v})), \\
\mathfrak{r} & =\mathfrak{r}^{*, \delta}(\theta, \mathfrak{c}, \zeta) .
\end{aligned}
$$


Moreover, it is standard to prove at this level the attainment of initial conditions and we have

$$
\boldsymbol{v}^{n}(0):=P_{1}^{n}\left(\boldsymbol{v}_{0}\right), \quad \mathfrak{c}(0):=\mathfrak{c}_{0}^{\varepsilon, \delta}, \quad e(0):=e^{\varepsilon, \delta} .
$$

5.4. Limit $\delta \rightarrow 0_{+}$. This is the key passage of the paper. We let $\delta \rightarrow 0_{+}$and consequently we remove the presence of the cut-off functions $T_{\delta}$ and $\mathcal{T}_{\delta}$. However, to do so we need to prove that the concentration are strictly positive uniformly. This is also the reason why we modify the definition of the entropy $s_{c}^{*}$. Hence, we denote the solution constructed in the previous subsection by the superscript $\delta$ and omit writing all other indexes. Moreover, the constant $C$ is again independent of the order of approximation.

First, we recall the minimum principle for the internal energy, that is preserved due to the strong convergence (5.76), i.e.,

$$
e^{\delta} \geq \delta \quad \text { almost everywhere in } Q .
$$

Next, we prove the same result also for the concetration $\mathfrak{c}^{\delta}$. Thus, setting

$$
u:=\min \left(0, c_{1}-\delta\right), \quad \boldsymbol{u}:=(u, 0, \ldots, 0)
$$

in (5.87) we deduce (we use the presence of the cut-off functions $\mathcal{T}_{\delta}$ and the fact that $\operatorname{div} \boldsymbol{v}^{\delta}=0$ )

$$
c_{1} \geq \delta
$$

almost everywhere in $Q$ and repeating the same procedure we can easily deduce that

$$
c_{i}^{\delta} \geq \delta \quad \text { almost everywhere in } Q \text { for all } i=1, \ldots, L .
$$

Moreover, setting

$$
u:=u \ell
$$

in (5.87) we can deduce with the help of (2.28) and (2.31) the following identity

$$
\left\langle\left(\mathfrak{c}^{\delta} \cdot \ell\right)_{, t}, u\right\rangle+\varepsilon\left(\nabla\left(\mathfrak{c}^{\delta} \cdot \ell\right), \nabla u\right)-\left(\left(\mathfrak{c}^{\delta} \cdot \ell\right) \boldsymbol{v}^{\delta}, \nabla u\right)=0 \quad \text { for all } u \in W^{1,2}(\Omega) \text { and a.a. } t \in(0, T) .
$$

Consequently, using the fact that

$$
\mathfrak{c}_{0}^{\delta} \cdot \ell \equiv 1 \quad \text { in } \Omega
$$

and that $\operatorname{div} \boldsymbol{v}^{\delta}=0$ we see that

$$
\mathfrak{c}^{\delta} \cdot \ell=1 \quad \text { almost everywhere in } Q,
$$

which together with (5.95) lead to

$$
\mathfrak{c}^{\delta} \in G \text { almost everywhere in } Q .
$$

Thus, it directly follows (5.96) that

$$
\sup _{t \in(0, T)}\left\|\mathfrak{c}^{\delta}(t)\right\|_{\infty} \leq L .
$$

Moreover, it also follows from (5.95), (5.96) and the definition of $\zeta^{*, \varepsilon, \delta}$ and $\zeta^{*, \varepsilon}$ that (assuming also that $\delta \leq 2)$

$$
\zeta^{\delta}=\zeta^{*, \varepsilon, \delta}\left(\mathfrak{c}^{\delta}\right)=\zeta^{*, \varepsilon}\left(\mathfrak{c}^{\delta}\right) \quad \text { a.e. in } Q .
$$

Next, for the first a priori estimate, we repeat the same procedure as in the preceding subsections. Thus, for the velocity field we have exactly in the same manner as before

$$
\begin{aligned}
\sup _{t \in(0, T)} & \left\|\boldsymbol{v}^{\delta}(t)\right\|_{2}^{2}+\int_{0}^{T}\left\|\boldsymbol{v}^{\delta}\right\|_{1, r}^{r}+\left\|\mathbf{S}^{\delta}\right\|_{r^{\prime}}^{r^{\prime}} d t \leq C, \\
& \int_{0}^{T}\left\|\boldsymbol{v}_{, t}^{\delta}\right\|_{2}^{2}+\left\|\boldsymbol{v}^{\delta}\right\|_{1,2 r}^{2 r}+\left\|\mathbf{S}^{\delta}\right\|_{2 r^{\prime}}^{2 r^{\prime}} d t \leq C(n) .
\end{aligned}
$$

Consequently, setting $u \equiv 1$ in (5.88) we get by using (5.99) and by (5.94) that

$$
\sup _{t \in(0, T)}\left\|e^{\delta}(t)\right\|_{1} \leq C .
$$

The crucial role however play the following estimates based on the entropy inequality. Note please, that now we heavily use the minimum principles (5.94) and (5.95) in order to guarantee 
that the test functions (in this case $1 / \theta^{\delta}$ and $\zeta^{\delta}$ ) belongs to the correct spaces. Hence, first we see that due to (5.94) we can set

$$
u:=\frac{1}{\theta^{\delta}}
$$

in (5.88) to obtain (note that $\operatorname{since} \operatorname{div} \boldsymbol{v}^{\delta}=0$ the convective term vanishes)

$$
\begin{aligned}
& \frac{d}{d t} \int_{\Omega} s_{e}^{*}\left(e^{\delta}\right) d x=-\varepsilon \int_{\Omega} \partial_{e^{2}}^{2} s_{e}^{*}\left(e^{\delta}\right)\left|\nabla e^{\delta}\right|^{2} d x+\int_{\Omega} \frac{\mathbf{S}^{\delta} \cdot \mathbf{D}\left(\boldsymbol{v}^{\delta}\right)}{\theta^{\delta}} d x \\
& \quad+\int_{\Omega} \mathcal{T}_{\delta}\left(\mathfrak{c}^{\delta}\right) T_{\delta}\left(e^{\delta}\right) \frac{\kappa\left(\mathfrak{c}^{\delta}, \theta^{\delta}\right)}{\left(\theta^{\delta}\right)^{2}}\left|\nabla \theta^{\delta}\right|^{2} d x-\left(\mathcal{T}_{\delta}\left(\mathfrak{c}^{\delta}\right) T_{\delta}\left(e^{\delta}\right) \nabla \zeta^{\delta}, \nabla \frac{1}{\theta^{\delta}} \otimes \mathfrak{m}\left(\mathfrak{c}^{\delta}, \theta^{\delta}\right)\right) .
\end{aligned}
$$

Similarly, setting $\boldsymbol{u}:=-\zeta^{\delta}$ in (5.87), which is a possible setting, we get (note that the convective term vanishes again)

$$
\begin{aligned}
& \frac{d}{d t} \int_{\Omega} s_{\mathfrak{c}}^{*, \varepsilon}\left(\mathfrak{c}^{\delta}\right) d x=\varepsilon\left(\nabla \mathfrak{c}^{\delta}, \nabla \zeta^{\delta}\right)-\left(\mathfrak{r}^{\delta}, \zeta^{\delta}\right) \\
& \quad+\left(\mathcal{T}_{\delta}\left(\mathfrak{c}^{\delta}\right) T_{\delta}\left(e^{\delta}\right)\left(M\left(\mathfrak{c}^{\delta}, \theta^{\delta}\right) \nabla \zeta^{\delta}+\nabla \frac{1}{\theta^{\delta}} \otimes \mathfrak{m}\left(\mathfrak{c}^{\delta}, \theta^{\delta}\right)\right), \nabla \zeta^{\delta}\right) .
\end{aligned}
$$

Thus, summing (5.102) and (5.103) we obtain the following identity

$$
\begin{array}{r}
\frac{d}{d t} \int_{\Omega} s_{e}^{*}\left(e^{\delta}\right)+s_{\mathfrak{c}}^{*, \varepsilon}\left(\mathfrak{c}^{\delta}\right) d x=-\varepsilon \int_{\Omega} \partial_{e^{2}}^{2} s_{e}^{*}\left(e^{\delta}\right)\left|\nabla e^{\delta}\right|^{2} d x+\varepsilon\left(\nabla \mathfrak{c}^{\delta}, \nabla \zeta^{\delta}\right) \\
+\int_{\Omega} \frac{\mathbf{S}^{\delta} \cdot \mathbf{D}\left(\boldsymbol{v}^{\delta}\right)}{\theta^{\delta}} d x+\int_{\Omega} \mathcal{T}_{\delta}\left(\mathfrak{c}^{\delta}\right) T_{\delta}\left(e^{\delta}\right) \frac{\kappa\left(\mathfrak{c}^{\delta}, \theta^{\delta}\right)}{\left(\theta^{\delta}\right)^{2}}\left|\nabla \theta^{\delta}\right|^{2} d x \\
+\left(\left(\mathcal{T}_{\delta}\left(\mathfrak{c}^{\delta}\right) T_{\delta}\left(e^{\delta}\right) M\left(\mathfrak{c}^{\delta}, \theta^{\delta}\right) \nabla \zeta^{\delta}, \nabla \zeta^{\delta}\right)-\left(\mathfrak{r}^{\delta}, \zeta^{\delta}\right)\right.
\end{array}
$$

First, we notice that all terms on the right hand side are nonnegative. Indeed, using (2.40), we get that

$$
-\varepsilon \int_{\Omega} \partial_{e^{2}}^{2} s_{e}^{*}\left(e^{\delta}\right)\left|\nabla e^{\delta}\right|^{2} d x \geq \varepsilon C_{1}\left\|\nabla \ln e^{\delta}\right\|_{2}^{2} .
$$

Next, it follows from (2.42) and (5.1) that

$$
\varepsilon\left(\nabla \mathfrak{c}^{\delta}, \nabla \zeta^{\delta}\right) \geq \varepsilon C^{-1}\left\|\nabla \mathfrak{c}^{\delta}\right\|_{2}^{2} .
$$

Moreover, the assumptions (A3)-(A4) directly imply nonnegativity of the third and fourth terms on the right hand side of (5.104). In addition, assuming (2.30), we see that

$$
\left(\mathcal{T}_{\delta}\left(\mathfrak{c}^{\delta}\right) T_{\delta}\left(e^{\delta}\right) M\left(\mathfrak{c}^{\delta}, \theta^{\delta}\right) \nabla \zeta^{\delta}, \nabla \zeta^{\delta}\right) \geq M_{1}\left\|\sqrt{\mathcal{T}_{\delta}\left(\mathfrak{c}^{\delta}\right) T_{\delta}\left(e^{\delta}\right)} P_{\ell} \nabla \zeta^{\delta}\right\|_{2}^{2} .
$$

Finally, from (2.28) and (5.92) we get that

$$
-\left(\mathfrak{r}^{\delta}, \zeta^{\delta}\right) \geq r_{1}\left\|\sqrt[q]{\mathcal{T}_{\delta}\left(\mathfrak{c}^{\delta}\right) T_{\delta}\left(e^{\delta}\right)} P_{\ell} \zeta^{\delta}\right\|_{q}^{q}
$$

Hence, substituting (5.105)-(5.108) into (5.104) and integrating with respect to $t \in(0, T)$, using the assumptions (2.38), the definition (5.1) and the a priori estimates (5.94), (5.97) and (5.101) we get

$$
\begin{array}{r}
\sup _{t \in(0, T)}\left(\left\|\ln e^{\delta}(t)\right\|_{1}+\varepsilon \max _{i=1, \ldots, L}\left\|\ln c_{i}^{\delta}(t)\right\|_{1}\right)+\varepsilon \int_{0}^{T}\left\|\nabla \mathfrak{c}^{\delta}\right\|^{2}+\left\|\nabla \ln \left(e^{\delta}\right)\right\|_{2}^{2} d t \\
+\int_{0}^{T}\left\|\sqrt[q]{\mathcal{T}_{\delta}\left(\mathfrak{c}^{\delta}\right) T_{\delta}\left(e^{\delta}\right)} P_{\ell} \zeta^{\delta}\right\|_{q}^{q}+\left\|\sqrt{\mathcal{T}_{\delta}\left(\mathfrak{c}^{\delta}\right) T_{\delta}\left(e^{\delta}\right)} P_{\ell} \nabla \zeta^{\delta}\right\|_{2}^{2} d t \\
\leq C\left(1+\varepsilon \max _{i=1, \ldots, L}\left\|\ln \left(c_{0}\right)_{i}\right\|_{1}\right) \leq C .
\end{array}
$$

Thus, setting $u:=e^{\delta}$ in (5.88), using (2.39), (5.100) and (5.109) we obtain

$$
\varepsilon \int_{0}^{T}\left\|\nabla e^{\delta}\right\|_{2}^{2} d t \leq C(n, \varepsilon)
$$


Next, we use the assumptions (A2), (A3) and the inequality (5.4) to deduce from (5.109) that (note that we use here the properties (2.32) and (2.35))

$$
\int_{0}^{T}\left\|\mathbf{q}_{\mathfrak{c}}^{\delta}\right\|_{2}^{2}+\left\|\mathbf{q}_{e}^{\delta}\right\|_{2}^{2}+\left\|\mathfrak{r}^{\delta}\right\|_{q^{\prime}}^{q^{\prime}}+\left\|\sqrt[q]{\mathcal{T}_{\delta}\left(\mathfrak{c}^{\delta}\right) T_{\delta}\left(e^{\delta}\right)} \zeta\right\|_{q}^{q} d t \leq C(\varepsilon, n)
$$

Consequently, using this estimate and the relations (5.48)-(5.88) and also the fact that the basis is smooth we obtain in the standard way that

$$
\left\|\mathfrak{c}_{, t}^{\delta}\right\|_{\left(L^{2}\left(0, T ; W^{1,2}\left(\Omega ; \mathbb{R}^{L}\right)\right) \cap L^{q}\left(0, T ; L^{q}\left(\Omega ; \mathbb{R}^{L}\right)\right)\right)^{*}}+\int_{0}^{T}\left\|\boldsymbol{v}_{, t}^{\delta}\right\|_{2}^{2}+\left\|e_{, t}^{\delta}\right\|_{-1,2}^{2} d t \leq C(\delta, n) .
$$

Thus, having (5.97), (5.99)-(5.101) and (5.109)-(5.112), we can extract a subsequence that we do not relabel and by using the Aubin-Lions lemma we get

$$
\begin{gathered}
\boldsymbol{v}^{\delta} \rightarrow \boldsymbol{v} \\
\boldsymbol{v}^{\delta} \rightarrow \boldsymbol{v} \\
\mathfrak{c}^{\delta} \rightarrow \mathfrak{c} \\
\mathfrak{c}_{, t}^{\delta} \rightarrow \mathfrak{c}_{, t} \\
\mathfrak{c}^{\delta} \rightarrow \mathfrak{c} \\
e^{\delta} \rightarrow e \\
e^{\delta} \rightarrow e \\
\mathbf{q}_{e}^{\delta} \rightarrow \mathbf{q}_{e} \\
\mathbf{q}_{\mathfrak{c}}^{\delta} \rightarrow \mathbf{q}_{\mathfrak{c}} \\
\mathbf{S}^{\delta} \rightarrow \mathbf{S} \\
\mathfrak{r}^{\delta} \rightarrow \mathfrak{r}
\end{gathered}
$$
weakly in $W^{1,2}\left(0, T ; V^{n}\right)$, strongly in $\mathcal{C}\left(0, T ; V^{n}\right)$, weakly in $L^{2}\left(0, T ; W^{1,2}\left(\Omega ; \mathbb{R}^{L}\right)\right)$, weakly in $\left(L^{2}\left(0, T ; W^{1,2}\left(\Omega ; \mathbb{R}^{L}\right)\right) \cap L^{q}\left(0, T ; L^{q}\left(\Omega ; \mathbb{R}^{L}\right)\right)\right)^{*}$, strongly in $L^{p}\left(0, T ; L^{p}\left(\Omega ; \mathbb{R}^{L}\right)\right)$ for all $p \in[1, \infty)$, weakly in $W^{1,2}\left(0, T ; W^{-1,2}(\Omega)\right) \cap L^{2}\left(0, T ; W^{1,2}(\Omega)\right)$, strongly in $L^{2}\left(0, T ; L^{2}(\Omega)\right)$,

The above convergence results allow us to let $\delta \rightarrow 0_{+}$in (5.86)-(5.88) to observe

$$
\begin{gathered}
\left(\boldsymbol{v}_{, t}, \boldsymbol{w}\right)-(\boldsymbol{v} \otimes \boldsymbol{v}, \nabla \boldsymbol{w})+(\mathbf{S}, \nabla \boldsymbol{w})=0 \quad \text { for all } \boldsymbol{w} \in V_{\mathrm{div}}^{n}, \\
\left\langle\mathfrak{c}_{, t}, \boldsymbol{u}\right\rangle+\varepsilon(\nabla \mathfrak{c}, \nabla \boldsymbol{u})-(\mathfrak{c} \otimes \boldsymbol{v}, \nabla \boldsymbol{u})-\left(\mathbf{q}_{\mathfrak{c}}, \nabla \boldsymbol{u}\right)=(\mathfrak{r}, \boldsymbol{u}) \\
\quad \text { for all } \boldsymbol{u} \in W^{1,2}\left(\Omega ; \mathbb{R}^{L}\right) \text { and a.a. } t \in(0, T), \\
\left\langle e_{, t}, u\right\rangle+\varepsilon(\nabla e, \nabla u)-(e \boldsymbol{v}, \nabla u)-\left(\mathbf{q}_{e}, \nabla u\right)=(\mathbf{S}, u \nabla \boldsymbol{v}) \\
\text { for all } u \in W^{1,2}(\Omega) \text { and a.a. } t \in(0, T) .
\end{gathered}
$$

Moreover, it is standard to prove at this level the attainment of initial conditions and we have

$$
\boldsymbol{v}(0)=P_{1}^{n}\left(\boldsymbol{v}_{0}\right), \quad \mathfrak{c}(0)=\mathfrak{c}_{0}^{\varepsilon}:=\frac{\mathfrak{c}_{0}}{1+\varepsilon L}+\frac{\varepsilon \ell}{1+\varepsilon L}, \quad e(0)=e_{0}^{\varepsilon}:=\min \left(\varepsilon^{-1}, e_{0}\right)+\varepsilon .
$$

Hence, to end this subsection, we need to show that

$$
\begin{aligned}
\mathbf{q}_{\mathfrak{c}} & =\mathbf{q}_{\mathfrak{c}}^{*}(\theta, \mathfrak{c}, \nabla \zeta, \nabla \theta), \\
\mathbf{q}_{e} & =\mathbf{q}_{e}^{*}(\theta, \mathfrak{c}, \nabla \zeta, \nabla \theta), \\
\mathbf{S} & =\mathbf{S}^{*}(\theta, \mathfrak{c}, \mathbf{D}(\boldsymbol{v})), \\
\mathfrak{r} & =\mathfrak{r}(\theta, \mathfrak{c}, \zeta),
\end{aligned}
$$

where

$$
\begin{aligned}
& \theta=\theta^{*}(e), \\
& \zeta=\zeta^{*, \varepsilon}(\mathfrak{c}) .
\end{aligned}
$$


First, due to the continuity of $\theta^{*}$ it directly follows from (5.118) and (5.119) that

$$
\begin{array}{ll}
\theta^{\delta} \rightarrow \theta & \text { weakly in } L^{2}\left(0, T ; W^{1,2}(\Omega)\right), \\
\theta^{\delta} \rightarrow \theta & \text { strongly in } L^{2}\left(0, T ; L^{2}(\Omega)\right),
\end{array}
$$

with $\theta$ given by (5.132). Consequently, due to the continuity of $\mathbf{S}^{*}$ we can use the strong and point-wise convergence results (5.117) and (5.135) to get (5.130). In order to identify the remaining limits, we first denote

$$
\begin{aligned}
& Q_{\delta_{0}}^{\delta}:=\left\{(x, t) \in Q ; \min \left(e^{\delta}(t, x), c_{1}^{\delta}(t, x), \ldots, c_{L}^{\delta}(t, x)\right) \leq 2 \delta_{0}\right\}, \\
& Q_{\delta_{0}}:=\left\{(x, t) \in Q ; \min \left(e(t, x), c_{1}(t, x), \ldots, c_{L}(t, x)\right) \leq 2 \delta_{0}\right\} .
\end{aligned}
$$

First, we see that for any fixed $\delta_{0}>0$ and for all $\delta \leq \delta_{0}$ it follows from (5.109) and (5.111) that

$$
\begin{aligned}
& \sup _{t \in(0, T)}\left(\left\|\ln e^{\delta}(t)\right\|_{1}+\varepsilon \max _{i=1, \ldots, L}\left\|\ln c_{i}^{\delta}(t)\right\|_{1}\right) \\
& \quad+\int_{0}^{T}\left\|\sqrt[q]{\mathcal{T}_{\delta_{0}}\left(\mathfrak{c}^{\delta}\right) T_{\delta_{0}}\left(e^{\delta}\right)} \zeta^{\delta}\right\|_{q}^{q}+\left\|\sqrt{\mathcal{T}_{\delta_{0}}\left(\mathfrak{c}^{\delta}\right) T_{\delta_{0}}\left(e^{\delta}\right)} P_{\ell} \nabla \zeta^{\delta}\right\|_{2}^{2} d t \leq C(\varepsilon, n) .
\end{aligned}
$$

Then using the Fatou lemma, weak-lower semicontinuity of norms, the definition of $\zeta^{\delta}$ and the convergence results $(5.115),(5.117),(5.118),(5.119)$ and (5.109) we get that

$$
\begin{aligned}
& \sup _{t \in(0, T)}\left(\|\ln e(t)\|_{1}+\varepsilon \max _{i=1, \ldots, L}\left\|\ln c_{i}(t)\right\|_{1}\right) \\
& \quad+\int_{0}^{T}\left\|\sqrt{\mathcal{T}_{\delta_{0}}(\mathfrak{c}) T_{\delta_{0}}(e)} \zeta\right\|_{q}^{q}+\left\|\sqrt{\mathcal{T}_{\delta_{0}}(\mathfrak{c}) T_{\delta_{0}}(e)} P_{\ell} \nabla \zeta\right\|_{2}^{2} d t \leq C(\varepsilon, n)
\end{aligned}
$$

with $\zeta$ given by (5.133). Next, it follows from (5.136) and (5.137) that

$$
\begin{aligned}
& \left|Q_{\delta_{0}}^{\delta}\right|:=\int_{Q_{\delta_{0}}^{\delta}} 1 d x d t \leq \int_{Q} \frac{\left|\ln e^{\delta}(t, x)\right|+\sum_{i=1}^{L}\left|\ln c_{i}^{\delta}(t, x)\right|}{\left|\ln \left(2 \delta_{0}\right)\right|} d x d t \leq \frac{C(\varepsilon, n)}{\left|\ln 2 \delta_{0}\right|}, \\
& \left|Q_{\delta_{0}}\right|:=\int_{Q_{\delta_{0}}} 1 d x d t \leq \int_{Q} \frac{|\ln e(t, x)|+\sum_{i=1}^{L}\left|\ln c_{i}(t, x)\right|}{\left|\ln \left(2 \delta_{0}\right)\right|} d x d t \leq \frac{C(\varepsilon, n)}{\left|\ln 2 \delta_{0}\right|}
\end{aligned}
$$

and consequently

$$
\left|Q_{0}\right|=0
$$

Since

$$
T_{\delta_{0}} \nearrow 1, \quad \mathcal{T}_{\delta_{0}} \nearrow 1, \quad \text { in } Q \backslash Q_{0} \text { for } \delta_{0} \rightarrow 0_{+},
$$

we can use (5.139) and the monotone convergence theorem to deduce from (5.137) that

$$
\sup _{t \in(0, T)}\left(\|\ln e(t)\|_{1}+\varepsilon \max _{i=1, \ldots, L}\left\|\ln c_{i}(t)\right\|_{1}\right)+\int_{0}^{T}\|\zeta\|_{q}^{q}+\left\|P_{\ell} \nabla \zeta\right\|_{2}^{2} d t \leq C(\varepsilon, n) .
$$

Finally, we show (5.128), (5.129) and (5.131). Thus, let $\boldsymbol{u} \in L^{\infty}\left(Q ; \mathbb{R}^{d}\right)$ be arbitrary, then

$$
\begin{aligned}
\lim _{\delta \rightarrow 0_{+}} \int_{0}^{T}\left(\mathbf{q}_{e}^{\delta}, \boldsymbol{u}\right) d t & =\lim _{\delta \rightarrow 0_{+}} \int_{0}^{T}\left(\left(\mathcal{T}_{\delta}\left(\mathfrak{c}^{\delta}\right) T_{\delta}\left(e^{\delta}\right)-\mathcal{T}_{\delta_{0}}\left(\mathfrak{c}^{\delta}\right) T_{\delta_{0}}\left(e^{\delta}\right)\right) \mathbf{q}_{e}^{*}\left(\theta^{\delta}, \mathfrak{c}^{\delta}, \nabla \zeta^{\delta}, \nabla \theta^{\delta}\right), \boldsymbol{u}\right) d t \\
& +\lim _{\delta \rightarrow 0_{+}} \int_{0}^{T}\left(\mathcal{T}_{\delta_{0}}\left(\mathfrak{c}^{\delta}\right) T_{\delta_{0}}\left(e^{\delta}\right) \mathbf{q}_{e}^{*}\left(\theta^{\delta}, \mathfrak{c}^{\delta}, \nabla \zeta^{\delta}, \nabla \theta^{\delta}\right), \boldsymbol{u}\right) d t=: I_{1}^{\delta_{0}}+I_{2}^{\delta_{0}} .
\end{aligned}
$$

Next, due to the linearity of $\mathbf{q}_{e}^{*}$ with respect to gradients and due to the presence of fixed (with respect to $\delta$ ) cut-off functions, we can use the weak convergence results (5.115) and (5.134), the continuity of $\zeta^{*, \varepsilon}$ and the strong convergence results (5.117) and (5.135) to easily identify the second limit as (using also (2.35))

$$
\left.I_{2}^{\delta_{0}}=\int_{0}^{T}\left(\mathcal{T}_{\delta_{0}}(\mathfrak{c}) T_{\delta_{0}}(e)\right) \mathbf{q}_{e}^{*}\left(\theta, \mathfrak{c}, \nabla \mathcal{P}_{\ell} \zeta, \nabla \theta\right), \boldsymbol{u}\right) d t
$$


Consequently, using the Lebesgue dominated convergence theorem, (5.134), (5.141), (5.139) and (5.140), we find that

$$
\lim _{\delta_{0} \rightarrow 0_{+}} I_{2}^{\delta_{0}}=\int_{0}^{T}\left(\mathbf{q}_{e}^{*}\left(\theta, \mathfrak{c}, \nabla \mathcal{P}_{\ell} \zeta, \nabla \theta\right), \boldsymbol{u}\right) d t .
$$

In addition, using (5.120) and the Hölder inequality, we get that

$$
\left|I_{1}^{\delta_{0}}\right| \leq C(\boldsymbol{u}, \varepsilon, n) \limsup _{\delta \rightarrow 0_{+}}\left|B_{\delta_{0}}^{\delta}\right|^{\frac{1}{2}},
$$

where the set $B_{\delta_{0}}^{\delta}$ is defined as

$$
B_{\delta_{0}}^{\delta}:=\left\{(x, t) \in Q ; \mathcal{T}_{\delta}\left(\mathfrak{c}^{\delta}\right) T_{\delta}\left(e^{\delta}\right) \neq \mathcal{T}_{\delta_{0}}\left(\mathfrak{c}^{\delta}\right) T_{\delta_{0}}\left(e^{\delta}\right)\right\} .
$$

Since, $B_{\delta_{0}}^{\delta} \subset\left(Q_{\delta_{0}}^{\delta} \cup\left\{(x, t) \in Q ; e^{\delta}>\frac{1}{\delta_{0}}\right\}\right)$, it directly follows from (5.119) and (5.138) that

$$
\limsup _{\delta_{0} \rightarrow 0_{+}}\left|I_{1}^{\delta_{0}}\right|=0
$$

and consequently it follows from (5.142) and the uniqueness of the weak limit that (5.129) holds. The same procedure then also leads to the identification of $\mathbf{q}_{\mathfrak{c}}$ and $\mathfrak{r}$, i.e., to (5.128) and (5.131).

In addition, by the Fatou lemma and the weak lower semicontinuity, we can also deduce from (5.104) that for almost all time $t \in(0, T)$

$$
\begin{aligned}
& \int_{\Omega} s_{e}^{*}(e(t))-s_{e}^{*}\left(e_{0}^{\varepsilon}\right)+s_{\mathfrak{c}}^{*, \varepsilon}(\mathfrak{c}(t))-s_{\mathfrak{c}}^{*, \varepsilon}\left(\mathfrak{c}_{0}^{\varepsilon}\right) d x \\
& \quad \geq \int_{0}^{t} \int_{\Omega} \frac{\mathbf{S} \cdot \mathbf{D}(\boldsymbol{v})}{\theta}+\frac{\kappa(\mathfrak{c}, \theta)}{\theta^{2}}|\nabla \theta|^{2} d x d \tau+\int_{0}^{t}(M(\mathfrak{c}, \theta) \nabla \zeta, \nabla \zeta)-(\mathfrak{r}, \zeta) d \tau .
\end{aligned}
$$

5.5. Limit $n \rightarrow \infty$ and $\varepsilon \rightarrow 0_{+}$. In this final subsection we prove the main theorem of the paper. Since, this limit passage follows step by step the proof of Theorem 3.1 we point out here only the essential differences. Thus, we set $\varepsilon:=\frac{1}{n}$ and then let $n \rightarrow \infty$ and denote by superscript $n$ the solution constructed in the preceding subsection. Note here that we already have

$$
\sup _{t \in(0, T)}\left\|e^{n}(t)\right\|_{1} \leq C
$$

and consequently it directly follows from (5.145) (see Section 4) that

$$
\sup _{t \in(0, T)}\left\|\ln e^{n}(t)\right\|_{1}+\int_{0}^{T}\left\|P_{\ell} \zeta^{n}\right\|_{1,2}^{2}+\left\|\zeta^{n}\right\|_{q}^{q} d t \leq C .
$$

But this is the starting point for all further estimates and also limit passage and one can repeat step by step the procedure in Section 4 to finish the proof of the main theorem. The only change is only the identification of the weak limit of $\zeta^{n}$, which heavily relies on the assumption (2.43) and we discuss it below. First, one can show (exactly as in Section 4) that for a subsequence

$$
\begin{aligned}
\mathfrak{c}^{n} & \rightarrow \mathfrak{c} \\
\mathcal{P}_{\ell} \zeta^{n} & \rightarrow \mathcal{P}_{\ell} \zeta \\
\zeta^{n} & \rightarrow \zeta
\end{aligned}
$$
strongly in $L^{2}\left(0, T ; L^{2}\left(\Omega ; \mathbb{R}^{L}\right)\right)$, weakly in $L^{2}\left(0, T ; W^{1,2}\left(\Omega ; \mathbb{R}^{L}\right)\right)$, weakly in $L^{q}\left(0, T ; L^{q}\left(\Omega ; \mathbb{R}^{L}\right)\right)$.

Hence, it remains to show that

$$
\zeta=\zeta^{*}(\mathfrak{c})
$$

and that

$$
\zeta^{n} \rightarrow \zeta
$$

stronlgly in $L^{1}\left(0, T ; L^{1}\left(\Omega ; \mathbb{R}^{L}\right)\right)$.

First, notice that it follows from (A7) and (5.148) that for arbitrary fixed $\delta>0$ (using also the fact that $\left.\mathfrak{c}^{n} \in G\right)$

$$
\mathcal{T}_{\delta}\left(\mathfrak{c}^{n}\right) \zeta^{n} \rightarrow \mathcal{T}_{\delta}(\mathfrak{c}) \zeta
$$$$
\text { strongly in } L^{1}\left(0, T ; L^{1}\left(\Omega ; \mathbb{R}^{L}\right)\right) \text {. }
$$ 
Thus, we see that all we need to show is that for any $K$ there exist $\delta>0$ such that for all $n$

$$
\left|Q_{\delta}^{n}\right| \leq \frac{1}{K}
$$

where

$$
Q_{\delta}^{n}:=\left\{(x, t) \in Q ; \min _{i=1, \ldots, L} c_{i}^{n} \leq \delta\right\} .
$$

Indeed, having (5.154), the relation (5.152) then directly follows from (5.150) and (5.153). To show (5.154) we first use (5.5) and (5.150) to obtain

$$
\int_{Q}\left|\zeta^{*}\left(\mathfrak{c}^{n}\right)\right|^{q} d x d t \leq C .
$$

Consequently, (5.154) directly follows from the assumption (2.43) and the proof of Theorem 3.2 is complete.

\section{REFERENCES}

[1] H. Abels and E. Feireisl: On a diffuse interface model for a two-phase flow of compressible viscous fluids. Indiana Univ. M., 57:659-697, 2007.

[2] M. Bulíček, E. Feireisl and J. Málek: A Navier-Stokes-Fourier system for incompressible fluids with temperature dependent material coefficients. Nonlinear Anal. Real World Appl., 10(2):992-1015, 2009.

[3] M. Bulíček, J. Málek and K. R. Rajagopal: Mathematical analysis of unsteady flows of fluids with pressure, shear-rate, and temperature dependent material moduli that slip at solid boundaries. SIAM J. Math. Anal., 41(2):665-707, 2009.

[4] M. Bulíček, P. Gwiazda, J. Málek and A. Świerczewska Gwiazda: On steady flows of an incompressible fluids with implicit power-law-like rheology. Adv. Calc. Var., 2:109-136, 2009.

[5] M. Bulíček, P. Gwiazda, J. Málek and A. Świerczewska Gwiazda: On Unsteady Flows of Implicitly Constituted Incompressible Fluids. SIAM J. Math. Anal., 44:2756-2801, 2012.

[6] M. Bulíček, J. Málek and K. R. Rajagopal: Mathematical results concerning unsteady flows of chemically reacting incompressible fluids. In J. C. Robinson and J. L. Rodrigo, editors, Partial Differential Equations and Fluid Mechanics. Cambridge University Press, 2009.

[7] L. Diening, M. Rǔžička and J. Wolf: Existence of weak solutions for unsteady motions of generalized Newtonian fluids. Ann. Sc. Norm. Super. Pisa Cl. Sci. (5), 9(1):1-46, 2010.

[8] W. E and P. Palffy-Muhorray: Phase separation in incompressible systems. Phys. Rev. E, 55:R3844-R3846, 1997.

[9] C. Eckart: The thermodynamics of irreversible processes II: fluid mixtures. Phys. Rev., 58:269-275, 1940.

[10] E. Feireisl: Dynamics of Viscous Compressible Fluids. Oxford university press, Oxford, 2003.

[11] E. Feireisl, H. Petzeltová and K. Trivisa: Multicomponent reactive flows: Global-in-time existence for large data. Comm. Pure Appl. Anal., 7:1017-1047, 2008.

[12] J. H. Ferziger and H. G. Kaper: Mathematical Theory of Transport Processes in Gases. North-Holland, Amsterdam, 1972.

[13] P. G. de Gennes: Dynamics of fluctuations and spinoidal fluctuations in polymer melts. J. Chem. Phys., 72:4756-4763, 1980.

[14] V. Giovangigli: Multicomponent Flow Modelling. Birkhäuser, Boston, 1999.

[15] M. Grmela: Why GENERIC? J. Non-Newt. Fluid Mech., 165:980-986, 2010.

[16] S. de Groot and P. Mazur: Nonequlibrium Thermodynamics. Dover, New York, 1984.

[17] J. Kim and J. Lowengrub: Phase field model and simulation of three-phases flows. Interfaces and Free Boundaries, 17:435-466, 2005.

[18] L. Magnarelli: Polarized mixtures with ionic tracers. J. Phys. A: Math. Theor., 42:1751-8113, 2009.

[19] J. Málek, J. Nečas, M. Rokyta and M. Růžička: Weak and measure-valued solutions to evolutionary PDEs, volume 13 of Applied Mathematics and Mathematical Computation. Chapman \& Hall, London, 1996.

[20] A. Mielke: A gradient structure for reaction-diffusion systems and for energy-drift-diffusion systems. Preprint WIAS no. 1485,Berlin 2010. Submited.

[21] A. Miranville and G. Schimperna: Nonisothermal phase separation based on a microforce balance. DCDS$B, 5: 753-768,2005$.

[22] J. W. Nunziato and E. K. Walsh: On ideal multiphase mixtures with chemical reactions and diffusion. Arch. Rat. Mech. Anal., 73:285-311, 1979.

[23] I. Prigogine: Thermodynamics of Irreversible Processes. Interscience Publishers, John Wiley \& Sons, New York, 1967.

[24] K. R. Rajagopal and L. Tao: Mechanics of Mixtures. World Scientific, Singapore, 1995.

[25] T. Roubíček: Incompressible ionized fluid mixtures: a non-Newtonian approach. IASME Transactions, 2:11901197, 2005.

[26] T. Roubíček: Incompressible ionized fluid mixtures. Cont. Mech. Thermodyn., 17:493-509, 2006. 
[27] T. Roubíček: Incompressible ionized non-Newtonian fluid mixtures. SIAM J. Math. Anal., 39:863-890, 2007.

[28] I. Samohýl: Application of Trusdell's model of mixtures to an ionic liquid mixture. Comp. Math. Appl., 53:182-197, 2007.

[29] H. C. Öttinger: Beyond Equilibrium Thermodynamics. Wiley - Interscience, Hoboken, New Jersey, 2005.

[30] H. C. Öttinger: Constraints in nonequlibrium thermodynamics: General framework and application to multicomponent diffusion. J. Chem. Phys., 130:114904, 2009.

Charles University, Faculty of Mathematics and Physics, Mathematical Institute, Sokolovská 83, 18675 Prague 8, Czech Republic

E-mail address: mbul8060@karlin.mff.cuni.cz

Charles University, Faculty of Mathematics and Physics, Mathematical Institute, Sokolovská 83, 18675 Prague 8, Czech Republid

E-mail address: havrda@karlin.mff.cuni.cz 